\title{
Computationally efficient forecasting procedures for Kuhn-Tucker consumer demand model systems: Application to residential energy consumption analysis
}

\author{
Abdul Rawoof Pinjari ${ }^{\mathrm{a}, *}$, Chandra Bhat ${ }^{\mathrm{b}}$ \\ ${ }^{a}$ Department of Civil Engineering, Centre for Infrastructure, Sustainable Transportation and Urban Planning (CiSTUP), Indian Institute of Science, \\ Sir C.V. Raman Road, Bangalore, 560012, India \\ ${ }^{\mathrm{b}}$ Dept of Civil, Architectural \& Environmental Engineering, The University of Texas at Austin1 University Station C1761, Austin, TX, 78712-0278, \\ USA
}

\section{A R T I C L E I N F O}

\section{Keywords:}

Discrete-continuous models

Kuhn-tucker consumer demand systems MDCEV model

Forecasting procedure

Residential energy consumption

Climate change impacts

\begin{abstract}
A B S T R A C T
This paper proposes simple and computationally efficient forecasting algorithms for random utility maximization-based multiple discrete-continuous (MDC) choice models with additively separable utility functions, such as the Multiple Discrete-Continuous Extreme Value (MDCEV) model. The algorithms build on simple yet insightful, analytical explorations with the Karush Kuhn-Tucker (KKT) conditions of optimality that shed new light on the properties of the models. The MDCEV model and the forecasting algorithms proposed in this paper are applied to a household-level energy consumption dataset to analyze residential energy consumption patterns in the United States. Further, simulation experiments are undertaken to assess the computational performance of the proposed and existing KT demand forecasting algorithms for a range of choice situations with small and large choice sets.
\end{abstract}

\section{Introduction}

Most choice modeling literature has focused on analyzing consumer demand situations that involve the choice of a single, discrete alternative from a set of choice alternatives that are mutually exclusive to (or perfect substitutes of) each other. In such situations, the consumer's choice of one alternative precludes the choice of other alternatives. However, in numerous situations, consumer choices may be characterized by "multiple discreteness," where consumers can potentially choose multiple alternatives, as opposed to a single alternative, because the choice alternatives are imperfect substitutes. Further, along with the discrete-choice decisions of which alternative(s) to choose, consumers typically make continuous quantity decisions on how much of each chosen alternative to consume. Such multiple discrete-continuous choice (MDC) situations are being increasingly recognized and modeled in the recent literature in transportation, marketing, and economics.

A variety of modeling frameworks have been used to analyze MDC choice situations. Among these, a particularly attractive approach is based on the classical microeconomic consumer theory of random utility maximization (RUM). Specifically, consumers are assumed to optimize a direct utility function $U(\mathbf{x})$ over a bundle of non-negative consumption quantities $\mathbf{x}=\left(x_{1}, \ldots, x_{k}, \ldots, x_{K}\right)$ subject to a linear budget constraint, as:

\footnotetext{
* Corresponding author.

E-mail addresses: abdul@iisc.ac.in (A.R. Pinjari), bhat@mail.utexas.edu (C. Bhat).
} 
In the above equation is a quasi-concave, increasing and continuously differentiable utility function with respect to the consumption quantity vector $\mathbf{x}, \mathbf{p}$ is the vector of unit prices $\left(p_{k}, k=1,2, \ldots, K\right)$ for all goods, and $E$ is the budget available for total expenditure. The consumption vector $\mathbf{x}$ may or may not include an outside good, which, when included, is a Hicksian composite good that represents all goods other than the $K-1$ inside goods of interest to the analyst. Typically, the outside good is treated as a numeraire with unit price, implying that the prices of all goods clubbed into this category do not influence the expenditure allocation among the inside goods (Deaton and Muelbauer, 1980). The form of the utility function governs the characteristics of the optimal consumption bundle resulting from the above utility maximization problem. A typical assumption made on the functional form is that the contribution to the utility $U(\mathbf{x})$ from the different goods is additively separable. That is, $U(\mathbf{x})=U\left(x_{1}\right)+U\left(x_{2}\right)+\ldots U\left(x_{k}\right)+\ldots+U\left(x_{K}\right)$. Another typical assumption is that the Hicksian composite good is considered essential in that it is always consumed by all the decisionmakers.

An increasingly popular approach for deriving the demand functions from the utility maximization problem in (1), especially in the context of modeling MDC choices, is based on the familiar Karush-Kuhn-Tucker (KKT) conditions of optimality. When the utility function is assumed to be randomly distributed over the population (from the analyst's perspective), the resulting KKT conditions are also stochastic in nature. Such randomly distributed KKT conditions provide a basis for deriving the likelihood expressions for observed consumption patterns. Due to the central role played by the KKT conditions, consumer demand models based on this approach are also called Kuhn-Tucker (KT) demand models.

The KT demand systems have been known for quite some time, dating back at least to the works of Hanemann (1978) and Wales and Woodland (1983). However, it is only in the past two decades that practical formulations of the KT demand system have appeared in the literature. Among these, the multiple discrete-continuous extreme value (MDCEV) model structure proposed by Bhat (2005, 2008) has gained substantial attention in the literature. The model is based on a translated constant elasticity of substitution (CES) utility function, whose random utility terms $\varepsilon_{k}(\forall k)$ enter the utility function as $U\left(x_{k}\right)=u\left(x_{k}\right) e^{\varepsilon_{k}}$, where $u\left(x_{k}\right)$ is the deterministic component of the utility from consuming good $k$ and $\varepsilon_{k}$ are specified as independent and identically distributed type- 1 extreme value. Due to a careful specification of the utility function and its stochastic terms, the MDCEV model offers an intuitive and clear interpretation of the utility function parameters, closed-form likelihood expressions, and subsumes several other KT demand systems in the literature as special cases.

Over the past decade, the basic MDCEV framework has been expanded in several directions, including (a) the incorporation of general error structures to allow flexible inter-alternative correlations (Pinjari and Bhat, 2010; Pinjari, 2011; Bhat et al., 2013), (b) incorporation of multiple budget constraints (Castro et al., 2012; Satomura et al., 2011; Pellegrini et al., 2021) and lower and upper bounds on consumption (Van Nostrand et al., 2013; Saxena et al., 2020), and (c) accommodation of flexible utility forms (Vasqez-Lavín and Hanemann, 2009; Bhat et al., 2015, 2020; Bhat, 2018; Palma and Hess, 2020), and the use of the MDC framework within an integrated choice and latent variable framework (Enam et al., 2017). The model and its variants have been applied in many empirical contexts, including individual activity participation and time-use (Bhat, 2005, 2008; Habib and Miller, 2009; Rajagopalan et al., 2009; Pinjari and Bhat 2010; Sikder and Pinjari, 2013; Calastri et al., 2017), household travel expenditures (Rajagopalan and Srinivasan, 2008; Ferdous et al., 2010), household vehicle ownership and usage forecasting (Ahn et al., 2007; Fang, 2008; Bhat et al., 2008; Pinjari et al., 2016), shippers' mode and port choices for freight infrastructure planning (Tapia et al., 2020), outdoor recreational demand (Phaneuf et al., 2000; von Haefen et al., 2004; von Haefen and Phaneuf, 2005), and grocery purchases (Kim et al., 2002).

Despite the many developments and applications, a simple and very quick forecasting procedure has not been available for the MDCEV and other KT demand model systems. On the other hand, since the end-goal of most model development and estimation is forecasting, policy evaluation, and/or welfare analysis, the development of a simple and easily applicable forecasting procedure is a critical issue in the application of KT demand model systems. The forecasting methods available prior to this work are either enumerative or iterative in nature, are not very accurate, and require long computation times.

In this paper, we propose computationally efficient ${ }^{1}$ forecasting algorithms for random utility maximization-based multiple discrete-continuous choice models with additively separable utility functions, such as the MDCEV model. The algorithms build on simple yet insightful, analytic explorations with the KKT conditions that shed new light on the properties of such models. For specific utility functional forms used in many MDCEV model applications, the proposed approach is non-iterative and results in analytically expressible consumption quantities once the chosen alternatives are known. Even with more general utility forms that fall within the class of additively separable utility functions, we employ the model properties presented in this paper to design efficient (albeit iterative) forecasting algorithms. Also, we formulate variants to the proposed algorithms that remain computationally efficient even in situations with large choice sets. Further, the insights gained from the analysis of the KKT conditions can be used to develop efficient forecasting procedures for other KT demand systems with additively separable utility functions.

As a demonstration of the effectiveness of the proposed algorithms, we present an application to analyze residential energy consumption patterns in the U.S., using household-level energy consumption data from the 2005 Residential Energy Consumption Survey (RECS) conducted by the Energy Information Administration (EIA). This application provides insights into the influence of household, house-related, and climatic factors on households' consumption patterns of different types of energy, including electricity, natural gas, fuel oil, and liquefied petroleum gas (LPG). Prediction exercises with the proposed algorithms and currently used algorithms highlight the significant computational efficiency of the proposed algorithms. In addition, we present simulation experiments to assess the

${ }^{1}$ We use the term efficient interchangeably with the term computationally efficient but not for statistical efficiency. 
computational performance of the proposed algorithms vis-à-vis existing algorithms in situations with large choice sets.

The remainder of the paper is organized as follows. The next section presents the challenge associated with forecasting with MDC choice models and describes the forecasting procedures used in the literature. Section 3 highlights some new properties of the MDC choice models with additively separable utility functions. Building on these properties, Section 4 presents new forecasting algorithms tailored for different types of utility specifications under different situations, ranging from small to large choice sets. In addition, a discussion is provided on how similar forecasting algorithms can be developed for other KT demand system models. Section 5 presents an application of the MDCEV model to analyze residential energy consumption patterns in the U.S., using the 2005 RECS data. Section 6 presents several prediction experiments with the RECS data as well as other simulated data to assess the computational performance of the proposed forecasting algorithms vis-à-vis existing approaches. In addition, this section includes hypothetical policy simulations to predict the impact of different climate change-related scenarios on residential energy consumption patterns. Section 7 summarizes and concludes the paper.

\section{Forecasting with KT demand model systems}

The MDCEV and other KT demand modeling systems are based on a resource allocation formulation. Specifically, it is assumed that consumers operate with a finite amount of available resources (i.e., a budget), such as time or money. Their decision-making mechanism is assumed to be driven by an allocation of the limited amount of resources to consume various goods/choice-alternatives to maximize the utility derived from consumption. Further, a stochastic utility framework is used to recognize the analyst's lack of awareness of factors affecting consumer decisions. In addition, a non-linear utility function is employed to incorporate important features of consumer behavior, including (1) the diminishing nature of marginal utility with increasing consumption (i.e., satiation effects), and (2) the possibility of consuming multiple goods as opposed to a single good. To summarize, the KT demand modeling frameworks are based on a stochastic (due to the stochastic utility framework), constrained (due to the budget constraint), non-linear (due to satiation effects) utility optimization formulation.

In most KT demand system models, the stochastic KKT first-order conditions of optimality form the basis for model estimation. Specifically, an assumption that stochasticity (or unobserved heterogeneity) is multivariate extreme value (MEV) distributed leads to closed-form consumption probability expressions (Bhat 2005; 2008; Pinjari 2011; von Haefen et al., 2004), facilitating a straightforward maximum likelihood estimation of the model parameters. Once the model parameters are estimated, forecasting or policy analysis exercises involve solving the stochastic, constrained, non-linear utility maximization problem for the optimal consumption quantities of each decision-maker. Unfortunately, there is no straightforward analytic solution to this problem. The typical approach is to adopt a constrained non-linear optimization procedure at each of several simulated values drawn from the distribution of unobserved heterogeneity. This constrained non-linear optimization procedure itself is based on either an enumerative technique or an iterative technique. The enumerative technique (used by Phaneuf et al., 2000) involves enumeration of all possible sets of alternatives that the decision-maker can potentially choose to consume. Specifically, if there are $K$ available choice alternatives, assuming not more than one essential Hicksian composite good, one can enumerate $2^{K}-1$ possible choice set solutions to the consumer's utility maximization problem. Clearly, such a brute-force method becomes computationally burdensome and impractical, even with a modest number of available choice alternatives/goods. Thus, for medium to large numbers of choice alternatives, an iterative optimization technique has to be used. As with any iterative technique, optimization begins with an initial solution (for consumptions) that is then improved in subsequent steps (or iterations) by moving along specific directions using the gradients of the utility functions, until a desired level of accuracy is reached. Most studies in the literature use off-the-shelf optimization programs (such as the constrained maximum likelihood library of GAUSS) to undertake such iterative optimization. However, the authors' experience with iterative methods of forecasting in prior research efforts indicates several problems, including long computation times and convergence issues.

More recently, von Haefen et al. (2004) proposed another iterative forecasting algorithm designed based on the insight that the optimal consumptions of all goods can be derived if the optimal consumption of the outside good is known. Specifically, conditional on the simulated values of unobserved heterogeneity (i.e., random utility terms), von Haefen et al. begin their iterations by setting the lower bound for the consumption of the outside good to zero and the upper bound to be equal to the budget. The average of the lower and upper bounds is used to obtain the initial estimate of the outside good consumption. Based on this, the amounts of consumption of all other inside goods are computed using the KKT conditions. Next, a new estimate of consumption of the outside good is obtained by subtracting the budget on the consumption of the inside goods from the total budget available. If this new estimate of the outside good is larger (smaller) than the earlier estimate, the earlier estimate becomes the new lower (upper) bound of consumption for the outside good, and the iterations continue until the difference between the lower and upper bounds is within an arbitrarily designated threshold. This numerical bisection iterative process relies on the strict concavity of the utility function. Further, to circumvent the need to perform predictions over the entire distribution of unobserved heterogeneity, von Haefen et al. condition on the observed choices. ${ }^{2}$ Based on "Monte Carlo experiments with low-dimensional choice sets", they indicate that, relative to the unconditional approach (of simulating the entire distribution of unobserved heterogeneity), the conditional approach requires about 1/3rd the simulations (of conditional unobserved heterogeneity) and time to produce stable estimates of mean consumptions and welfare measures. Overall, this

\footnotetext{
${ }^{2}$ To do so, they simulate the unobserved heterogeneity that corresponds to the observed choices in the data (i.e., they simulate the error terms for each individual so as to replicate the observed consumptions of the individual). Using these simulated values of conditional unobserved heterogeneity, they apply the numerical bisection algorithm to perform predictions for the policy case and then compute the welfare change from the base case to policy case.
} 
combination of the numerical bisection algorithm with the conditional approach is clever and clearly more efficient than using a generic optimization procedure with the unconditional approach. However, the numerical bisection algorithm is still iterative and can involve a substantial amount of time. At the same time, in many situations, the estimated model needs to be applied to data outside the estimation sample, in which case the conditional approach cannot be used. For instance, in the travel demand field, models are estimated with an intent to apply them for predicting the activity-travel patterns in the external (to estimation sample) data representing the study area population. Besides, estimated models are often applied on holdout samples to validate the model. This implies that the iterative numerical bisection algorithm has to be applied using the unconditional approach, which could further increase computation time. The point is that there is a computational benefit to using a non-iterative optimization procedure rather than an iterative procedure, which can then be used with the conditional approach when possible or with the unconditional approach if needed. Further, and more importantly, the von Haefen et al. algorithm is applicable only in the case with the presence of an outside good. To be more precise, their approach can be applied only if the analyst knows apriori at least one good chosen by the consumer. In situations with an outside good, it is already known that the outside good is one of the consumed alternatives. However, in situations with no outside good, their approach does not provide any lead to the analyst on which alternative is consumed (or not consumed), a critical prerequisite for obtaining the consumption forecasts.

\section{The MDCEV model: structure and properties}

This section draws from Bhat (2008) to briefly discuss the structure of the MDCEV model (Section 3.1) and derives some fundamental properties of the model (Section 3.2) that will form the basis for formulating the forecasting algorithm.

\subsection{Model structure}

Consider the following additively separable utility function as in Bhat (2008):

$$
U(\boldsymbol{t})=\frac{1}{\alpha_{1}} \psi_{1} t_{1}{ }^{\alpha_{1}}+\sum_{k=2}^{K} \frac{\gamma_{k}}{\alpha_{k}} \psi_{k}\left\{\left(\frac{t_{k}}{\gamma_{k}}+1\right)^{\alpha_{k}}-1\right\} ; \psi_{k}>0,0 \leq \alpha_{k} \leq 1, \gamma_{k}>0
$$

In the above expression, $U(t)$ is the total utility accrued from consuming $t$ (a $K x 1$ vector with non-negative consumption quantities $\left.t_{k} ; k=1,2, \ldots, K\right)$ amount of the $K$ alternatives available to the consumer. The $\psi_{k}$ terms $(k=1,2, \ldots, K)$, labeled as baseline utility parameters, represent the marginal utility of one unit of consumption of alternative $k$ at the point of zero consumption for that alternative. Through the $\psi_{k}$ terms, the impact of observed and unobserved alternative attributes, decision-maker attributes, and the choice environment attributes may be introduced as $\psi_{k}=\exp \left(\beta^{\prime} z_{k}+\varepsilon_{k}\right)$, where $z_{k}$ contains the observed attributes and $\varepsilon_{k}$ is a random disturbance capturing the unobserved factors. The $\alpha_{k}$ terms $(k=1,2, \ldots, K)$, labeled satiation parameters $\left(0<\alpha_{k} \leq 1\right)$, capture satiation effects by reducing the marginal utility accrued from each unit of additional consumption of alternative $k .^{3}$ The $\gamma_{k}$ terms $(k=2,3, \ldots, K)$, labeled translation parameters, play a similar role of satiation as that of $\alpha_{k}$ terms, and an additional role of translating the indifference curves associated with the utility function to allow corner solutions (i.e., accommodate the possibility that decision-makers may not consume all alternatives). As it can be observed, there is no $\gamma_{k}$ term for the first alternative, for it is assumed to be an essential Hicksian composite good (or outside good or essential good) that is always consumed (hence there is no need for a corner solution).

The optimal consumptions from the utility maximization problem can be found by forming the Lagrangian and applying KKT conditions. The Lagrangian function for the utility function in (2) is:

$$
L=\frac{1}{\alpha_{1}} \psi_{1}\left(x_{1}\right)^{\alpha_{1}}+\sum_{k=2}^{K} \frac{\gamma_{k}}{\alpha_{k}} \psi_{k}\left\{\left(\frac{x_{k}}{\gamma_{k}}+1\right)^{\alpha_{k}}-1\right\}-\lambda\left[\sum_{k=1}^{K} p_{k} x_{k}-E\right]
$$

where $\lambda$ is the Lagrange multiplier associated with the budget constraint. The KKT first-order conditions for the optimal consumptions $\left(x_{k}^{*} ; k=1,2, \ldots, K\right)$ are given by:

$$
\begin{aligned}
& \frac{\psi_{1}}{p_{1}}\left(x_{1}^{*}\right)^{\alpha_{1}-1}-\lambda=0 \text { since } x_{1}^{*}>0 \\
& \frac{\psi_{k}}{p_{k}}\left(\frac{x_{k}^{*}}{\gamma_{k}}+1\right)^{\alpha_{k}-1}-\lambda=0 \text { if } x_{k}^{*}>0,(\mathrm{k}=2,3, \ldots, \mathrm{K}) \\
& \frac{\psi_{k}}{p_{k}}\left(\frac{x_{k}^{*}}{\gamma_{k}}+1\right)^{\alpha_{k}-1}-\lambda<0 \text { if } x_{k}^{*}=0,(\mathrm{k}=2,3, \ldots, \mathrm{K})
\end{aligned}
$$

As indicated earlier, these stochastic KKT conditions form the basis for model estimation. Next, using these same stochastic KKT

\footnotetext{
${ }^{3}$ In theory, $\alpha_{k}$ can take negative values. But the condition $\alpha_{k} \geq 0$ provides stability in empirical estimations (Bhat, 2008). Further, $\alpha_{k}=1 \forall k$ is a special case implying linear utility with respect to consumption for all choice alternatives. In such a case, the utility maximizing consumer will choose only one choice alternative with the highest price-normalized marginal utility. For the model to allow multiple discreteness, at least one of the $\alpha_{k}$ values should be less than 1 . When $\alpha_{k} \rightarrow 0 \forall k$, the utility function takes a logarithmic, $\gamma$-profile form shown later in Eq. (20).
} 
conditions, we derive a few properties of the MDCEV model that can be exploited to develop a computationally efficient forecasting algorithm.

\subsection{Properties of the MDCEV model}

Property 1. The price-normalized baseline utility of a chosen good is always greater than that of a good that is not chosen.

$$
\left(\frac{\psi_{i}}{p_{i}}\right)>\left(\frac{\psi_{j}}{p_{j}}\right) \text { if } \mathrm{i} \text { is a chosen good and } \mathrm{j} \text { is not a chosen good }
$$

Proof. The KKT conditions in (4) can be rewritten as:

$$
\begin{aligned}
& \frac{\psi_{1}}{p_{1}}\left(x_{1}^{*}\right)^{\alpha_{1}-1}=\lambda \\
& \frac{\psi_{k}}{p_{k}}\left(\frac{x_{k}^{*}}{\gamma_{k}}+1\right)^{\alpha_{k}-1}=\lambda \text { if } x_{k}^{*}>0,(\mathrm{k}=2,3, \ldots, \mathrm{K}) \text { (i.e., for all chosen goods) } \\
& \frac{\psi_{k}}{p_{k}}<\lambda \text { if } x_{k}^{*}=0,(\mathrm{k}=2,3, \ldots, \mathrm{K}) \text { (i.e., for all goods that are not chosen) }
\end{aligned}
$$

The above KKT conditions can further be rewritten as:

$$
\begin{aligned}
& \left(\frac{\psi_{k}}{p_{k}}\right)=\left(\frac{x_{k}^{*}}{\gamma_{k}}+1\right)^{1-\alpha_{k}}\left(\frac{\psi_{1}}{p_{1}}\right)\left(x_{1}^{*}\right)^{\alpha_{1}-1}, \quad \text { if } x_{k}^{*}>0,(k=2,3, \ldots, K) \\
& \left(\frac{\psi_{k}}{p_{k}}\right)<\left(\frac{\psi_{1}}{p_{1}}\right)\left(x_{1}^{*}\right)^{\alpha_{1}-1}, \quad \text { if } x_{k}^{*}=0,(k=2,3, \ldots, K)
\end{aligned}
$$

Now, consider two alternatives, ' $\mathrm{i}$ ' and ' $\mathrm{j}$ ', of which ' $\mathrm{i}$ ' is chosen and ' $\mathrm{j}$ ' is not chosen by a consumer. For that consumer, the above KKT conditions for alternatives 'i' and 'j' can be written as:

$$
\begin{aligned}
& \left(\frac{\psi_{i}}{p_{i}}\right)=\left(\frac{x_{i}^{*}}{\gamma_{i}}+1\right)^{1-\alpha_{i}}\left(\frac{\psi_{1}}{p_{1}}\right)\left(x_{1}^{*}\right)^{\alpha_{1}-1}, \text { and } \\
& \left(\frac{\psi_{j}}{p_{j}}\right)<\left(\frac{\psi_{1}}{p_{1}}\right)\left(x_{1}^{*}\right)^{\alpha_{1}-1}
\end{aligned}
$$

Further, since $\left(\frac{x_{j}^{*}}{\gamma_{j}}+1\right)^{1-\alpha_{j}}$ is always greater than 1 , one can write the following inequality:

$$
\left(\frac{\psi_{j}}{p_{j}}\right)<\left(\frac{\psi_{1}}{p_{1}}\right)\left(x_{1}^{*}\right)^{\alpha_{1}-1}<\left(\frac{x_{i}^{*}}{\gamma_{i}}+1\right)^{1-\alpha_{i}}\left(\frac{\psi_{1}}{p_{1}}\right)\left(x_{1}^{*}\right)^{\alpha_{1}-1}
$$

As one can observe, the third term in the above inequality is nothing but $\left(\frac{\psi_{i}}{p_{i}}\right)$, and the second term is $\lambda$. Thus, one can rewrite the inequality in (9) as:

$$
\left(\frac{\psi_{j}}{p_{j}}\right)<\lambda<\left(\frac{\psi_{i}}{p_{i}}\right)
$$

The above inequality implies a fundamental property of the MDCEV model that $\left(\frac{\psi_{i}}{p_{i}}\right)>\left(\frac{\psi_{j}}{p_{j}}\right)$. In other words, the price-normalized baseline utility of a chosen good is always greater than that of a good that is not chosen.

Corollary 1.1. It naturally follows from the property above that when all the $K$ alternatives/goods available to a consumer are arranged in descending order of their price-normalized baseline utility values (with the outside good being the first in the order), and if it is known that the number of chosen alternatives is $M$, then one can easily identify the chosen alternatives as the first $M$ alternatives in the arrangement. ${ }^{4}$

\footnotetext{
${ }^{4}$ Note that the converse of this property may not always hold true. That is, given the price-normalized utilities of two alternatives, one cannot say with certainty if one or both of the alternatives are chosen.
} 
Corollary 1.2. The Lagrange multiplier of the consumer's utility maximization problem (i.e., the marginal utility at optimal consumption) is always greater than the price-normalized utility of any not-chosen good, but less than that of any chosen good. It naturally follows from this property that $\lambda$ is greater than the highest price-normalized baseline utility among the not-chosen goods but less than the lowest price-normalized baseline utility among the chosen goods.

Property 2. When all the satiation parameters $\left(\alpha_{k}\right)$ are equal, and if the corner solutions are known (i.e., if the chosen and non-chosen alternatives are known), the Lagrange multiplier of the utility maximization, as well as the continuous optimal consumption choices of the chosen goods, can be expressed in an analytic form.

Proof: Using the first and second KKT conditions in (6), and assuming without loss of generality that the first M goods are chosen, one can express the optimal consumptions as:

$$
\begin{gathered}
x_{1}^{*}=\left(\lambda \frac{p_{1}}{\psi_{1}}\right)^{\frac{1}{\alpha_{1}-1}}, \text { and } \\
x_{k}^{*}=\left\{\left(\lambda \frac{p_{k}}{\psi_{k}}\right)^{\frac{1}{\alpha_{k}-1}}-1\right\} \gamma_{k} ; \forall k=(2,3, \ldots, M)^{5}
\end{gathered}
$$

Using the above expressions, the budget constraint can be written as: ${ }^{6}$

$$
p_{1}\left(\lambda \frac{p_{1}}{\psi_{1}}\right)^{\frac{1}{\alpha_{1}-1}}+\sum_{k=2}^{M} p_{k}\left\{\left(\lambda \frac{p_{k}}{\psi_{k}}\right)^{\frac{1}{a_{k}-1}}-1\right\} \gamma_{k}=E
$$

From the above equation, and assuming that all satiation $\left(\alpha_{k}\right)$ parameters as equal to $\alpha$, the Lagrange multiplier $\lambda$ can be expressed analytically as:

$$
\lambda=\left(\frac{E+\sum_{k=2}^{M} p_{k} \gamma_{k}}{p_{1}\left(\frac{\psi_{1}}{p_{1}}\right)^{\frac{1}{1-\alpha}}+\sum_{k=2}^{M} p_{k} \gamma_{k}\left(\frac{\psi_{k}}{p_{k}}\right)^{\frac{1}{1-\alpha}}}\right)^{\alpha-1}
$$

The above expression for $\lambda$ can be substituted back into the equations in (11) to obtain the following analytic expressions for optimal consumptions of the chosen goods:

$$
\begin{aligned}
& x_{1}^{*}=\frac{\left(\frac{\psi_{1}}{p_{1}}\right)^{\frac{1}{1-\alpha}}\left(E+\sum_{k=2}^{M} p_{k} \gamma_{k}\right)}{p_{1}\left(\frac{\psi_{1}}{p_{1}}\right)^{\frac{1}{1-\alpha}}+\sum_{k=2}^{M} p_{k} \gamma_{k}\left(\frac{\psi_{k}}{p_{k}}\right)^{\frac{1}{1-\alpha}}} \\
& x_{k}^{*}=\left(\frac{\left(\frac{\psi_{k}}{p_{k}}\right)^{\frac{1}{1-\alpha}}\left(E+\sum_{k=2}^{M} p_{k} \gamma_{k}\right)}{p_{1}\left(\frac{\psi_{1}}{p_{1}}\right)^{\frac{1}{1-\alpha}}+\sum_{k=2}^{M} p_{k} \gamma_{k}\left(\frac{\psi_{k}}{p_{k}}\right)^{\frac{1}{1-\alpha}}}-1\right) \gamma_{k} ; \forall k=(2,3, \ldots, M)
\end{aligned}
$$

The reader will note here that the expressions in Equations (13)-(15) contain terms corresponding to the consumed (i.e., chosen) goods only.

\section{Efficient forecasting algorithms for MDCEV and other KT demand models}

In this section, using the properties discussed in the preceding section, we propose efficient forecasting algorithms for the MDCEV model and other KT demand systems. Section 4.1 presents a non-iterative forecasting algorithm for the MDCEV model with the following utility function:

$$
U(\mathbf{x})=\frac{1}{\alpha} \exp \left(\beta^{\prime} z_{1}+\varepsilon_{1}\right)\left(x_{1}\right)^{\alpha}+\sum_{k=2}^{K} \frac{\gamma_{k}}{\alpha} \exp \left(\beta^{\prime} z_{k}+\varepsilon_{k}\right)\left\{\left(\frac{x_{k}}{\gamma_{k}}+1\right)^{\alpha}-1\right\},
$$

As indicated in Bhat (2008), this is a $\gamma$ - profile utility function in that the $\gamma_{k}$ parameters are different across the choice alternatives, but the satiation $\left(\alpha_{k}\right)$ parameters are constrained to be equal (to $\alpha$ ) across all choice alternatives. Section 4.2 presents a similar (but iterative) algorithm for more general utility functions that allow for different $\alpha_{k}$ parameters across choice alternatives. Section 4.3

\footnotetext{
${ }^{5}$ Although this expression involves a subtraction, it is not possible to obtain negative predictions. One can verify this by applying the KKT condition inequality: $\lambda<\left(\psi_{k} / p_{k}\right) \forall k \in$ chosen alternatives\} to see that the second expression always provides positive predictions.

${ }^{6}$ The expression on the left side of this budget constraint equation is a monotonically decreasing function of $\lambda$. This property will be useful later.
} 
presents variants to the proposed algorithms for situations with large choice sets. Section 4.4 discusses how such efficient forecasting algorithms can be designed for other KT demand systems in the literature with additively separable utility functions.

\subsection{Forecasting algorithm for the MDCEV model with $\gamma$-profile utility functions: an incremental enumeration method}

The proposed algorithm comprises four basic steps as outlined below.

- Step 0: Assume that only the outside good is chosen. Let the number of chosen goods $M=1$.

- Step 1: Given the input data $\left(z_{k}, p_{k}\right)$, model parameters $\left(\beta, \gamma_{k}, \alpha\right)$, and the simulated error term $\left(\varepsilon_{k}\right)$ draws, compute the pricenormalized baseline utility values $\left(\psi_{k} / p_{k}\right)$ for all alternatives.

- Step 2: Arrange all the $K$ alternatives available to the consumer in the descending order of their price-normalized baseline utility values (with the outside good in the first place).

- Step 3: To find the chosen alternatives, loop over the above-ordered list:

o Compute the value of $\lambda$ using Equation (13).

o If $\mathrm{M}$ is the last alternative or if $\lambda>\left(\psi_{M+1} / p_{M+1}\right)$ (i.e., if $\lambda>$ the price-normalized baseline utility of alternative in position $\left.\mathrm{M}+1\right)$,

o The first $M$ alternatives in the above ordering are the chosen alternatives

o Exit loop.

o Else, $\mathrm{M}=M+1$ and return to the loop.

- Step 4: Compute the optimal consumptions for the chosen alternatives using Equations (14) and (15). For non-chosen alternatives, set the optimal consumptions as zero.

The algorithm outlined above can be applied a large number of times with different simulated values of the $\varepsilon_{k}$ terms to sufficiently cover the simulated distribution of unobserved heterogeneity (i.e., the $\varepsilon_{k}$ terms) and obtain the distributions of the consumption forecasts. Alternatively, if the observed consumption choices are available, one can apply the algorithm with simulated values of conditional (on observed choices) unobserved heterogeneity as in von Haefen et al. (2004).

To apply the above algorithm for situations without an outside good, minor modifications would be needed. Specifically, instead of starting with the outside good as the chosen good (i.e., instead of step 0), after step 2 designate the alternative with the highest price normalized baseline utility as the first chosen good. The rest of the algorithm would follow the same steps as in the algorithm outlined above. In step 3, however, the following expression should be used for the Lagrange multiplier $(\lambda)$ instead of that in Equation (13):

$$
\lambda=\left(\frac{E+\sum_{k=1}^{M} p_{k} \gamma_{k}}{\sum_{k=1}^{M} p_{k} \gamma_{k}\left(\frac{\psi_{k}}{p_{k}}\right)^{\frac{1}{1-\alpha}}}\right)^{\alpha-1}
$$

and in step 4, the following expression should be used for the optimal consumption of all chosen alternatives; instead of the expressions in Equations (14) and (15):

$$
x_{k}^{*}=\left(\frac{\left(\frac{\psi_{k}}{p_{k}}\right)^{\frac{1}{1-\alpha}}\left(E+\sum_{k=1}^{M} p_{k} \gamma_{k}\right)}{\sum_{k=1}^{M} p_{k} \gamma_{k}\left(\frac{\psi_{k}}{p_{k}}\right)^{\frac{1}{1-\alpha}}}-1\right) \gamma_{k}
$$

\subsubsection{Intuitive interpretation of the algorithm}

The proposed algorithm builds on the insight from corollary 1.1 that if the number of chosen alternatives is known, one can easily identify the chosen alternatives by arranging the price-normalized baseline utility values in descending order. Subsequently, one can compute the optimal consumptions of the chosen alternatives using Equations (14) and (15). The only issue, however, is that the number of chosen alternatives is unknown apriori. To find this out, the algorithm begins with an assumption that only one alternative (i.e., the outside good) is chosen and verifies this assumption by examining the KKT conditions (i.e., if $\lambda>\left(\psi_{M+1} / p_{M+1}\right)$ ) for other (assumed to be) non-chosen goods. If the KKT conditions are met, the search for additional chosen alternatives stops. Else, at least the next alternative (in the order of the price-normalized baseline utilities) has to be among the chosen alternatives. Then, the KKT conditions are verified again by assuming that the next alternative is among the chosen alternatives. These basic steps are repeated until either the KKT conditions are met, or the assumed number of chosen alternatives reaches the maximum number $(K)$.

Note that the KKT conditions are essentially replaced by a single condition equivalent to verifying if $\lambda$ is greater than the highest price-normalized baseline utility among the not-chosen goods (that is, if $\lambda>\left(\psi_{M+1} / p_{M+1}\right)$ ). To understand this better, recall from (6) that verifying the KKT conditions in this algorithm is equivalent to verifying the condition $\lambda>\left(\psi_{k} / p_{k}\right)$ for all goods that are assumed to have not been chosen. Obviously, checking if $\lambda$ is greater than the highest price-normalized baseline utility of all goods assumed to have not been chosen is a more efficient way of doing so (see corollary 1.2).

The proposed algorithm involves enumeration of the choice baskets in a computationally efficient fashion. In fact, the algorithm begins with identifying a single alternative (outside good) that may be chosen. If the KKT conditions are not met for this choice basket, 
the algorithm identifies a two-alternative choice basket and so on till the number of chosen alternatives is determined. Thus, the number of times the algorithm enumerates choice baskets is equal to the number of chosen alternatives in the optimal consumption portfolio, which is at most equal to (but many times less than) the total number of available alternatives $(K)$. Thus, we label this algorithm an 'incremental enumeration algorithm'.

Another feature of the algorithm is that it is non-iterative, which makes it much more efficient compared to iterative approaches. Further, coding the algorithm using vector and matrix notation in a matrix programming language significantly reduces the computational burden even with a large number of choice alternatives and observations. Also, due to the convenient $\gamma-$ profile utility specification, the algorithm is accurate with no room for inaccuracy (unlike the existing iterative procedures discussed earlier), as it uses analytic expressions for the optimal consumption computations.

In summary, the proposed algorithm is simple and efficient. The only limitation of this algorithm is that it is designed to be used with the $\gamma$ - profile utility specification that restricts the $\alpha_{k}$ parameters of all choice alternatives to be equal. Admittedly, the $\gamma-$ profile utility specification is not the most general form within the class of additively separable utility functions. However, as indicated by Bhat (2008), both the $\gamma_{k}$ and $\alpha_{k}$ parameters serve the role of allowing differential satiation effects across the choice alternatives. Due to the overlapping roles played by these parameters, attempts to estimate utility functions that allow both the $\gamma_{k}$ and $\alpha_{k}$ parameters to vary across alternatives may lead to severe empirical identification issues and estimation breakdowns. For these reasons and given the ease of forecasting with the proposed algorithm, we suggest an estimation of the $\gamma$ - profileutility function. Nevertheless, the insights obtained from the properties discussed in the preceding section (and parts of this algorithm) can be used to design an efficient (albeit iterative) algorithm for cases when $\alpha_{k}$ parameters vary across alternatives (i.e., for $\alpha$ - profile utility functions), as discussed next.

\subsection{Forecasting algorithm for the MDCEV model with $\alpha$-Profile utility functions: incremental enumeration combined with bisection over the $\lambda$-space}

Let $\widehat{\lambda}$ and $\widehat{E}$ be estimates of $\lambda$ (the Lagrange multiplier) and $E$ (the budget amount), respectively, and let $\delta_{\lambda}$ and $\delta_{E}$ be the tolerance values (for estimating $\lambda$ and E, respectively) which can be as small as desired. Let $\hat{\lambda}_{L}$ and $\hat{\lambda}_{U}$ be the lower and upper bounds of $\lambda$. Based on the budget constraint in (12), define $\widehat{E}$ (the estimate of $E$ ) as a function of $\widehat{\lambda}$ (estimate of $\lambda$ ) as:

$$
\widehat{E}=p_{1}\left(\hat{\lambda} \frac{p_{1}}{\psi_{1}}\right)^{\frac{1}{\alpha_{1}-1}}+\sum_{k=2}^{M} p_{k}\left\{\left(\hat{\lambda} \frac{p_{k}}{\psi_{k}}\right)^{\frac{1}{\alpha_{k}-1}}-1\right\} \gamma_{k}
$$

The proposed algorithm comprises six basic steps as outlined below.

- Step 0: Assume that only the outside good is chosen. Let the number of chosen goods $M=1$.

- Step 1: Given the input data $\left(z_{k}, p_{k}\right)$, model parameters $\left(\beta, \gamma_{k}, \alpha_{k}\right)$ and the simulated error term $\left(\varepsilon_{k}\right)$ draws, compute the pricenormalized baseline utility values $\left(\exp \left(\beta^{\prime} z_{k}+\varepsilon_{k}\right) / p_{k}\right)$ for all alternatives. Arrange all the $K$ alternatives available to the consumer in descending order of their price-normalized baseline utility values (with the outside good in the first place, if there is an outside good).

- Step 2: Let $\widehat{\lambda}=\frac{\psi_{M+1}}{p_{M+1}}$, the price-normalized baseline utility of the alternative in position $M+1$. Substitute $\widehat{\lambda}$ into Equation (17) to obtain an estimate $\widehat{E}$ of $E$.

- Step 3:

If $\widehat{E}<E$

Go to step 4.

Else, if $\widehat{E}>E$

$\lambda_{L}=\frac{\psi_{M+1}}{p_{M+1}}$ and $\lambda_{U}=\frac{\psi_{M}}{p_{M}}$ (because $\frac{\psi_{M+1}}{p_{M+1}}<\lambda<\frac{\psi_{M}}{p_{M}}$ ).

Go to step 5 to estimate $\lambda$ via numerical bisection.

\section{- Step 4:}

$M=M+1$.

If $M<K$

Go to step 2.

Else, if $M=K$

$\lambda_{L}=0$ and $\lambda_{U}=\frac{\psi_{K}}{p_{K}}$ (because $0<\lambda<\frac{\psi_{K}}{p_{K}}$ ).

Go to step 5 to estimate $\lambda$ via numerical bisection.

\section{- Step 5:}

Step 5.1: Let $\widehat{\lambda}=\left(\lambda_{L}+\lambda_{U}\right) / 2$ and use Equation (17) to obtain an estimate $\widehat{E}$ of $E$.

Step 5.2: If $\left(\left|\lambda_{L}-\lambda_{U}\right| \leq \delta_{\lambda}\right.$ or $\left.|\widehat{E}-E| \leq \delta_{E}\right)$

\footnotetext{
${ }^{7}$ Strictly speaking, our proposed algorithm does have iterations in steps 2-4. However, in the spirit of the term "iterative" used in general for numerically based computationally intensive iterations, we call our approach non-iterative since the overall algorithm is analytical (as opposed to being numerical) in nature.
} 
Go to step 6 .

Else, if $\widehat{E}<E$

Update the upper bound of $\lambda$ as $\lambda_{U}=\left(\lambda_{L}+\lambda_{U}\right) / 2$, and go to step 5.1.

Else, if $\widehat{E}>E$

Update the lower bound of $\lambda$ as $\lambda_{L}=\left(\lambda_{L}+\lambda_{U}\right) / 2$, and go to step 5.1.

- Step 6: Compute the optimal consumptions of the first $M$ alternatives in the above descending order using equations in (11). Set the consumptions of other alternatives as zero and stop.

As can be observed, the above algorithm is similar to the algorithm in Section 4.1 in that the choice alternatives are arranged in descending order by the price-normalized baseline utility values. Further, the algorithm begins with an assumption that only one alternative is chosen (i.e., $M=1$ ) and builds on the following insights:

1. The value of the Lagrange multiplier $\lambda$ is greater than the highest price-normalized baseline utility among the not-chosen goods but less than the lowest price-normalized baseline utility among the chosen goods (corollary 1.2).

2. The left side of the budget constraint Equation (12) is a monotonically decreasing function of $\lambda$. Thus, in Equation (17), the value of $\widehat{E}$ increases monotonically as the value of $\widehat{\lambda}$ decreases.

Based on these insights, as indicated in step 2, the algorithm starts with an estimate $\hat{\lambda}$ of $\lambda$ as the price-normalized baseline utility of the alternative in the $(M+1)^{\text {th }}$ position in the above descending order and a corresponding estimate $\widehat{E}$ of $E$. Then step 3 verifies if the estimated amount of budget $\widehat{E}$ is less than the actual amount of budget $E$ available to the decision-maker. If so, the number of chosen alternatives $M$ is increased by 1 (see step 4), and the value of $\widehat{\lambda}$ is updated to the price-normalized baseline utility of the next alternative in the arrangement (see step 2). This process is repeated until either $\widehat{E}$ exceeds $E$ (step 3 ) or $M=K$ (step 4). At this point, the chosen alternatives are known (as the first $M$ alternatives in the descending order of price-normalized utility values). Further, an upper bound (lowest price-normalized baseline utility among the chosen alternatives) and a lower bound (highest price-normalized baseline utility among the not-chosen alternatives, or zero) are also available for $\lambda$. Given these bounds for $\lambda$ and the property that $\widehat{E}$ decreases monotonically with $\hat{\lambda}$, step 5 follows a simple numerical bisection to arrive at the final estimate for $\lambda$ that accurately estimates $E$. This is the value of $\lambda$ that satisfies the KKT conditions of optimal consumption. Thus, using this value of $\lambda$, one can use the KKT Equations in (11) to obtain the optimal consumptions.

The above algorithm is generic enough to be used for MDCEV models with either $\gamma$-profile utility functions or $\alpha$-profile utility functions. Replacing the numerical bisection procedure to compute $\lambda$ (i.e., step 5) in the above algorithm by an analytic formula for $\lambda$ (from Equation (14)) will result in a more efficient forecasting algorithm for the $\gamma$-profile model, which is equivalent to the algorithm proposed in Section 4.1. In other words, the difference between the forecasting algorithms proposed in sections 4.1 and 4.2 is in the way $\lambda$ is computed.

The algorithm presented in this section is similar to the von Haefen et al. algorithm in that both the algorithms use a numerical bisection approach. However, while von Haefen et al. employ bisection over the consumption (or expenditures)-space to arrive at the optimal consumptions, we first use incremental enumeration (as in Section 4.1) to identify the chosen alternatives and then employ numerical bisection over the $\lambda$-space to arrive at the optimal consumptions. We exploit the properties discussed in Section 3 to arrange the alternatives based on the price-normalized baseline utility values. This arrangement helps in quickly identifying the chosen alternatives. Thus, in situations with no essential good (where there is no apriori knowledge of any chosen good), this arrangement becomes important. On the other hand, the von Haefen et al. algorithm critically depends on the knowledge of a chosen good, but provides no way to determine a chosen alternative when there is no essential good in the set of choice alternatives. ${ }^{8}$

\subsection{Variants of the proposed forecasting algorithms for situations with large choice sets}

Recall from the discussion in Section 4.1.1 that the proposed algorithm for $\gamma$-profile utility functions in Section 4.1 is enumerative in nature. The number of times the algorithm enumerates the choice baskets is equal to the number of chosen alternatives in the optimal consumption portfolio. Even in the context of $\alpha$ - profile utility functions, the algorithm proposed in Section 4.2 enumerates the choice baskets as many times as the number of chosen alternatives. Thus, one may argue that the number of enumerations may become too large in situations with large number of chosen alternatives. To avoid such large number of enumerative steps, the proposed algorithms can be modified as discussed below.

\subsubsection{Bisection-based enumeration algorithm for $\gamma$-profile utility functions and large choice sets}

For choice situations with large choice sets and $\gamma$-profile utility functions, the algorithm proposed in Section 4.1 can be modified by replacing the step-by-step enumeration with a bisection-based enumeration (over the space of choice alternatives). Specifically, the

\footnotetext{
${ }^{8}$ This is not to say that the insights from the von Haefen et al. algorithm cannot be used in situations without at least one essential good. Once the alternatives are arranged in the descending order of the price-marginalized baseline utility values, one could employ numerical bisection over the consumption space (or expenditures space) for the first good in the arrangement to obtain the optimal consumption forecasts.
} 
algorithm begins with an assumption that the number of chosen alternatives, $M$ is an integer midway between the lower bound value (one) and an upper bound value $K$ (i.e., between the first alternative and the last alternative in the descending order of the baseline utility values). Subsequently, based on the lambda value computed for the corresponding $M$, either the lower bound or the upper bound is updated to an integer midway between the previous lower and upper bounds. This process continues until convergence is reached on the number of chosen alternatives $(M)$, and then the optimal consumptions are computed. Thus, the proposed modifications allow the algorithm to quickly determine the chosen alternatives without the need to enumerate each (and every) chosen alternative. The detailed steps of the algorithm are provided below.

- Step 0: Let lower bound for $M, M_{L}=1$, let upper bound for $M, M_{U}=K$.

- Step 1: Arrange all $K$ alternatives available to the consumer in the descending order of their price-normalized baseline utility values (with the outside good in the first place).

- Step 2: Let $M=\left\lfloor\left(M_{L}+M_{U}\right) / 2\right\rfloor$ (i.e., an integer midway between $M_{L}$ and $\left.M_{U}\right)$.

Compute the value of $\lambda$ using equation (13).

- Step 3:

If $\lambda>\frac{\psi_{M}}{p_{M}}$ (i.e., the price-normalized baseline utility of alternative in position $M$ )

Update upper bound $M_{U}=M-1$.

Else, if $\lambda<\frac{\psi_{M}}{p_{M}}$ (i.e., the price-normalized baseline utility of alternative in position $M$ ).

Update lower bound $M_{L}=M$.

- Step 4: Compute $M=\left\lfloor\left(M_{L}+M_{U}\right) / 2\right\rfloor$ (i.e., an integer midway between $M_{L}$ and $M_{U}$ ).

Compute the value of $\lambda$ using equation (13).

- Step 5:

If $\left(M_{L}<M\right)$, go to step 3 .

Else, if $\left(M_{L}=M\right)$ and if $\lambda>\frac{\psi_{M+1}}{p_{M+1}}$

Set $M=M_{L}$.

Else, if $\left(M_{L}=M\right)$ and if $\lambda<\frac{\psi_{M+1}}{p_{M+1}}$

Set $M=M_{L}+1$.

- Step 6: Compute the optimal consumptions using equations (14) and (15) for the first $M$ goods (in the arrangement of step 1). Set the consumptions of other goods as zero and stop.

4.3.2. Bisection (over the $\lambda$-space) algorithm for $\alpha$-profile utility functions and large choice sets

In the case of $\alpha$-profile utility functions, the algorithm proposed in Section 4.2 can be modified by avoiding the enumerative steps (i. e., steps 3 and 4 of the algorithm in section 4.2) and directly employing a bisection-based iteration over the $\lambda$-space (as in step 5 of section 4.2). The modified algorithm is presented below.

- Step 0: Assume that only the outside good is chosen and let the number of chosen goods $M=1$.

- Step 1: Arrange all the $K$ alternatives available to the consumer in descending order of their price-normalized baseline utility values (with the outside good in the first place).

- Step 2: Let $\lambda_{L}=0$ and $\lambda_{U}=\frac{\psi_{1}}{p_{1}}$, the price-normalized baseline utility of the alternative in the 1 st position.

- Step 3:

Step 3.1: Let $\widehat{\lambda}=\left(\lambda_{L}+\lambda_{U}\right) / 2$, and use Equation (17) to obtain an estimate $\widehat{E}$ of $E$.

- Step 3.2:

If $\left(\left|\lambda_{L}-\lambda_{U}\right| \leq \delta_{\lambda}\right.$ or $\left.|\widehat{E}-E| \leq \delta_{E}\right)$

Go to step 4.

Else, if $\widehat{E}<E$

Update the upper bound of $\lambda$ as $\lambda_{U}=\left(\lambda_{L}+\lambda_{U}\right) / 2$, and go to step 3.1.

Else, if $\widehat{E}>E$

Update the lower bound of $\lambda$ as $\lambda_{L}=\left(\lambda_{L}+\lambda_{U}\right) / 2$, and go to step 3.1.

- Step 4: Use KKT equations in (11) to compute the optimal consumptions of all alternatives. Reset the negative consumptions obtained from these equations to zero and stop.

The reader will note that this algorithm is much more similar to the von Haefen, 2003 algorithm than the algorithm in Section 4.2, which combines enumeration of choice alternatives with numerical bisection over the $\lambda$-space. The only difference is that while this algorithm employs bisection over the $\lambda$-space, the von Haefen et al. algorithm employs bisection over the consumption space. Thus, one can expect both the algorithms to provide similar computational performance.

\subsection{Generalization of the forecasting algorithms for other KKT demand model systems}

The algorithms developed above are tailored to the MDCEV model. However, similar properties as in Section 3 can be derived, and 
similar forecasting algorithms can be developed for other KKT demand model systems in the literature with non-linear and additively separable utility functions. Specifically, the basic algorithmic approach remains the same, except that some modifications may be needed depending on the form of the utility function employed in different KKT demand systems, as discussed below.

- In step 1 of both the algorithms, all the $K$ available alternatives have to be arranged in the descending order based on their pricenormalized marginal utility value at zero consumption. In fact, a general property of most KT demand systems used in the literature (with additively separable utility functions) is that the price-normalized marginal utility at zero consumption of a chosen alternative is always greater than that of a non-chosen alternative. This property can be proved for different forms of additively separable utility functions used in the literature by following the proof of Property 1 in Section 3.1.

- Similar to the above modification, in all other steps of both the algorithms, replace the price-normalized baseline utility with the corresponding price-normalized marginal utility measure at zero consumption.

- Similarly, for the algorithms in Section 4.1 and 4.3.1 for $\gamma$-profile utility functions, the analytic formula for Lagrange multiplier $(\lambda)$ will depend on the form of the utility function. The formulae for optimal consumptions (conditional on the knowledge of the consumed alternatives) will also depend on the form of the utility function. These formulae can be derived in a straightforward fashion following the discourse in Section 3.

In summary, the proposed algorithms are fairly generic in their approach and can be easily modified to be used with different forms of non-linear and additively separable utility functions.

\section{Empirical analysis of residential energy consumption patterns}

In this and the next section, we estimate a model of residential energy consumption and demonstrate the computational effectiveness and practical value of our proposed forecasting algorithms by applying them to study a variety of energy policy scenarios.

The interest in the subject of residential energy demand analysis dates back to the 1970s, reportedly beginning with the first oil crisis. This topic has gained renewed attention in recent years, due to at least three reasons. First, growing concerns regarding depleting fossil fuel resources, deteriorating environmental quality, and potentially adverse climate changes have highlighted the need for sustainable energy production and efficient energy consumption practices. Second, the residential sector is responsible for a significant share of total national-level energy consumption in many countries. For example, in the United States, the residential sector accounted for $22 \%$ of the total energy consumption during the year 2008 (EIA, 2009). Thus, the analysis of residential energy consumption patterns is essential for energy planning, pricing, and policy decision-making. Third, price fluctuations, energy policies, and market conditions may result in notable welfare consequences and equity/distributional impacts. For example, Cashin and McGranahan (2006) report that low-income households are likely to be impacted more than high-income households due to a rise in energy prices. Thus, it is important to understand energy consumption patterns in the context of price variations and policy impacts.

Section 5.1 provides an overview of residential energy consumption studies in the past. Section 5.2 describes the RECS data used in this study, and Section 5.3 presents and discusses the MDCEV model estimation results.

\subsection{Brief overview of the residential energy consumption literature}

Residential energy demand analysis was initiated by the pioneering work of Houthakker (1951) on British urban electricity consumption. Since then, and especially after the oil price shocks in the 1970s, numerous studies have examined energy consumption in the residential sector, using aggregate-level econometric demand techniques (e.g., Clements and Madlener, 1999; Narayan and Smith, 2005) or disaggregate, household-level, econometric techniques (Bhattacharyya and Timilsina, 2009). The focus of this paper is on disaggregate-household level econometric modeling techniques.

From a modeling perspective, several econometric studies use single-equation log-linear models that estimate the amount of a fuel used conditional upon a household choosing to use that fuel (e.g., Filippini and Pachauri, 2004). The approach is to regress the natural logarithm of energy consumption as a function of price, income, climate, and other variables influencing demand. Sometimes, log-log (or double-log) models, in which the explanatory variables (price, income, etc.) also take a logarithmic form along with the dependent variable, are also used (Dale et al., 2009). Log-linear and log-log models are easy to estimate and simple to interpret. For example, the coefficient of the log-price variable in a log-log model can be directly interpreted as the price elasticity of demand. However, these models have been criticized for the lack of a sound behavioral/theoretical foundation (Madlener, 1996). Another stream of studies uses the transcendental logarithmic (or translog) functional forms proposed by Christensen et al. (1973). The translog specification includes quadratic forms of the log-price and log-income variables in addition to first-order terms in a log-log specification. This specification comes with additional flexibility relative to the log-linear or log-log models, but there is also the potential problem of obtaining unintuitive/unstable parameter (and elasticity) estimates (Madlener, 1996). Further, one cannot use single-equation regression models (whether log-linear, log-log, or translog) to analyze households' consumption of multiple types of fuel simultaneously. Another limitation is that none of these approaches can be used to model the "discrete" choices households make on the type(s) of energy/fuel to use. On the other hand, a common feature of household energy consumption data (as will be discussed in a subsequent section) is that several households consume multiple types, but not necessarily all types, of energy available to them. This choice process can be viewed as a result of multiple discrete-continuous choices, where households make discrete choices of "whether to use" and continuous choices of "how much to use" for each type of energy available in the market.

Dubin and McFadden (1984) pioneered the joint analysis of the discrete choice of appliance holdings (choice of heating technology) 
and the continuous choice of energy consumption by explicitly accounting for the self-selection effects due to unobserved factors affecting both the discrete and continuous choices. Mansur et al. (2008) use this approach for simultaneous analysis of households' energy/fuel type and usage choices, while Nesbakken (2001) uses the approach for simultaneous analysis of the fuel type and usage choices for space heating. In such earlier studies, the discrete choice alternatives correspond to possible combinations of fuel types such as electricity only, electricity and natural gas, electricity and fuel oil, and electricity and other fuels. The continuous choice component includes the amount of consumption of each type of fuel, conditional upon the choice of fuel type combination (with self-selection terms included in the continuous choice model to account for the endogeneity of discrete choices). However, these two-step estimation procedures are generally inefficient and are also not based on a unified utility-maximizing theory of multiple discreteness. Rather, they are based on exploding the fuel type combinations to generate a choice set suitable for modeling in a traditional single discrete choice framework. Even at moderate sizes of the elemental fuel type alternatives, this approach of exploding the elemental alternatives starts to generate a large number of combination alternatives for the single discrete choice (for example, 4 elemental fuel type alternatives will generate 15 combination choices of fuel types for the single discrete choice). The result is that sample sizes for estimation of such models need to be relatively large to obtain enough households who choose each combination. On the other hand, multiple discrete-continuous KT demand systems handle such situations from a unified microeconomic principle of the choice of multiple alternatives and offer an efficient framework for estimation. ${ }^{9}$ In this paper, we employ the MDCEV model in which a unified utility maximization approach for multiple discreteness is adopted to simultaneously model the "whether to consume" and "how much to consume" decisions for all types of energy.

\subsection{Data}

The data used in this study was obtained from the 2005 Residential Energy Consumption Survey (RECS) conducted by the Energy Information Administration (EIA) of the United States. The RECS collected a variety of information on the use of energy for a sample of 4382 households in housing units statistically selected to represent the 111.1 million housing units in the U.S. The information collected in this survey included: (1) The sources/types of energy used (electricity, natural gas, fuel oil, liquid petroleum gas (LPG), kerosene, and wood) and the quantity of annual consumptions and corresponding expenditures for each source/type of energy, (2) The end-uses of energy (cooking, washing/drying, heating, cooling, etc.), (3) The physical characteristics of the housing unit, (4) Information on household appliances such as space heating and cooling equipment, and (5) The demographic characteristics of the household. The information was collected using a combination of the following survey administration methods: (a) in-person interviews with householders of sampled housing units, (b) mail questionnaires or in-person/telephone interviews with rental agents of sampled rental units where some or all energy costs were included in the rent, and (c) mail questionnaires from energy suppliers who provided actual energy consumption and expenditure data for the sampled housing unit. In addition to the above-mentioned information, each household's geographic location information was collected by the EIA but not released to the public due to confidentiality reasons (only Census region/division information and rural/urban classifications were made available in the public use microdata). However, EIA utilized the location information to obtain weather information from the National Oceanic and Atmospheric Administration. Thus, climate variables, such as annual heating degree days and annual cooling degree days (which are described in the next section), are available in the RECS data. Further details about the survey as well as the public-use microdata used for this study are available at the following EIA website: http://www.eia.doe.gov/emeu/recs/. A notable downside of this data is that information on gasoline usage for household travel purposes was not collected in this survey.

Among the 4382 household records in the RECS data, several records did not contain information on the consumption of different types of energy (especially LPG consumption). Those records were supplemented by EIA with imputed consumption values and expenditures. Such imputed information, although useful for aggregate-level analysis purposes, can be inaccurate and potentially influence the model estimates. Hence, only 2473 records with actual reported information on energy consumption and expenditures were used for model estimation. Of these 2473 households, all households consumed electricity, $59.2 \%$ (or 1465 households) consumed natural gas, $7.3 \%$ (180 households) consumed fuel oil, and 6.3\% (155 households) consumed LPG. ${ }^{10}$ It is worth noting here that very few households indicated kerosene and wood consumption. Hence, the kerosene and wood alternatives were not included in the analysis.

Table 1 presents more details on the energy consumption patterns in the estimation sample (see the first set of rows in the table). As

\footnotetext{
${ }^{9}$ Some discrete-continuous energy demand studies (e.g., Vaage, 2000) follow Hanemann's (1984) approach in which the discrete and continuous choices are derived theoretically from the same utility maximization problem. But this approach does not accommodate the usage of multiple types of energy. Other previous studies (e.g., Labanderia and Labeaga, 2006) have analyzed the multi-fuel/energy usage patterns of household by employing a quadratic extension of the almost ideal demand system (AIDS) of Deaton and Muellbauer (1980) as proposed by Banks et al. (1997). The AIDS system is based on a unified utility maximization principle for simultaneously modeling the consumption of multiple types of energy/fuels. However, the AIDS system assumes that all types of energy are consumed by all households. Such an assumption precludes an analysis of the discrete choices faced by households on the type(s) of energy to consume.

${ }^{10}$ The implied energy consumption patterns of the 2473 households (without weighting) were not very different from the implied consumption patterns of the 111.1 million US housing units (as obtained by expanding the original 4382 household records using weights). The only difference of relevance was in the percentage of households consuming LPG as an energy source. We chose to estimate the model with the unweighted sample of 2473 households because individual household imputations in the 4382 -household sample may be quite different from reality, even if the aggregate consumption patterns are not very different between the 2 473-household sample and the weighted 4 382- household sample.
} 
can be observed, 709 out of the 2473 households (i.e., 28.67\%) consumed only electricity and no other type of fuel. All other households (i.e., $71.33 \%$ in the sample) consumed multiple types of energy (i.e., at least one other type of energy than electricity). Further, no household chose both the natural gas and LPG alternatives, indicating that these two sources of energy are perfect substitutes. In fact, all 1465 households consuming natural gas belong to the category of 1779 households that had a natural gas pipe connection to their housing unit, while all 155 LPG consuming households did not have a natural gas pipe connection to their housing unit.

The average annual household expenditure and average consumption in millions of British Thermal Units (or MBTU) in each type of energy are reported in the second set of rows in Table 1. To this data on annual energy expenditures, an additional "outside good" expenditure variable was appended (see the last row in the second set of rows). The expenditure for the "outside good" for each household was computed by subtracting the household's total annual energy expenditures (i.e., expenditures on all four types of energy - electricity, natural gas, fuel oil, and LPG) from the annual household income. Thus, for analysis purposes, households are assumed to operate with their income as a budget constraint (see Equation (3)) and allocate income to consume different types of energy and to the outside good. The outside good includes other expenses as well as savings.

The unit prices for each type of energy are in the third set of rows. ${ }^{11}$ It can be observed that electricity is the most expensive fuel (for consumers), while natural gas is the least expensive fuel. The unit price for the outside good is set to unity. The next three sets of rows in the table provide descriptive information on the household, housing unit, and household residential location variables (in that order) used in the model. For continuous variables, the average value of that variable in the data is reported. For categorical/dummy variables, the number (and percentage) of households in the data is reported. For example, the average annual household income in the data is close to $\$ 50 \mathrm{k}$. About $30 \%$ of the households belong to the low-income category, while $23 \%$ belong to the high-income category. Similar interpretations hold for the other household-related variables.

The climate variable group includes two variables: the annual heating degree days (HDD) and the annual cooling degree days (CDD). The HDD variable is a surrogate measure of how cold a location is over a year, relative to a base temperature of $65^{\circ}$ Fahrenheit. For each location, it is computed as the difference between the average daily temperature and $65^{\circ}$ Fahrenheit (if the average daily temperature is less than $65^{\circ}$ Fahrenheit) summed over the 365 days in the year. Similarly, the annual cooling degree days (CDD) variable is computed as a measure of how hot a location is over a year (relative to a base temperature of $65^{\circ}$ Fahrenheit). The use of these variables in the model allows us to study the influence of climate factors on energy consumption patterns.

\subsection{Model estimation results}

The choice alternatives in the MDCEV model include the four types of energy (electricity, natural gas, fuel oil, and LPG) and a numeraire "outside good". We considered various estimable forms of the general utility function in Equation (2) proposed by Bhat (2008). The following form of the utility function provided the best fit to the current empirical data:

$$
U(\boldsymbol{e})=\psi_{o} \ln e_{o}+\psi_{e} \ln \left(\frac{e_{e}}{p_{e}}\right)+\gamma_{n} \psi_{n} \ln \left(\frac{e_{n}}{\gamma_{n} p_{n}}+1\right)+\gamma_{f} \psi_{f} \ln \left(\frac{e_{f}}{\gamma_{f} p_{f}}+1\right)+\gamma_{l} \psi_{l} \ln \left(\frac{e_{l}}{\gamma_{l} p_{l}}+1\right)
$$

In the above utility equation, on the right-hand side, the first term $\left(\psi_{o} \ln e_{o}\right)$ corresponds to the utility contribution of the expenditure $e_{o}$ to the outside good, the second term corresponds to the utility contribution due to the consumption of electricity (where $e_{e} / p_{e}$ is the consumption amount of electricity), the third, fourth and fifth terms correspond to the utility contributions due to the consumption of natural gas, fuel oil, and LPG, respectively. The subscripts, $o, e, n, f$, and $l$ used in the utility expression represent the choice alternatives of outside good, electricity, natural gas, fuel oil, and LPG, respectively.

The above utility form is obtained by constraining all the $\alpha_{k}$ terms (for $k=1,2,3,4,5$ ) in Equation (2) to be equal to zero (see Bhat, 2008). We used this utility form because initial estimations revealed that the $\alpha_{k}$ parameter estimates were not statistically different from zero. Further, there is no $\gamma_{k}$ term corresponding to the outside good and electricity categories because all households in the estimation sample allocated some non-zero amount of their income to these categories. The baseline marginal utility terms $\left(\psi_{k}\right)$ are specified as $\psi_{k}=\exp \left(\beta^{\prime} z_{k}+\varepsilon_{k}\right)$, where $z_{k}$ contains the observed factors (such as household characteristics, housing unit characteristics, location attributes, and climate variables) and $\varepsilon_{k}$ captures the unobserved factors influencing energy consumption decisions. Further, for identification purposes, the $\psi_{o}$ parameter corresponding to the outside good is specified as $\exp \left(\varepsilon_{o}\right)$, without any observed variables $z_{k}$ (i.e., the outside good acts as the base alternative in the model specification).

Table 2 presents the model estimates. The baseline preference constants (i.e., constants in $\psi_{k}$ functions) do not have any substantive interpretations but capture generic tendencies to consume each type of energy as well as accommodate the range of the continuous variables in the model. However, the positive baseline preference constant for electricity (relative to the constants for other energy

\footnotetext{
11 Given the data on energy consumption and expenditures, one could potentially compute the unit price values for each type of energy consumed by each household (as expenditure divided by consumption). However, since energy prices tend to vary with consumption levels (due to blockpricing), the unit price values computed in such a fashion would be endogenous to consumption levels. Further, for any household, it is possible to compute the unit prices only for those types of energy that the household consumed, but not for non-chosen energy types. Thus, instead of computing unit price values separately for each household from the data, we used the aggregate-level unit price values given by the EIA. These values, obtained from the EIA website at the following link, vary by census divisions: http://www.eia.doe.gov/emeu/recs/recs2005/hc2005_tables/ c\&e/pdf/tableus7.pdf.
} 
Table 1

Descriptive statistics of the RECS data.

\begin{tabular}{ll}
\hline Variable & Value \\
\hline Number (\%) of households consuming & \\
Electricity only & $709(28.67 \%)$ \\
Electricity and Natural Gas & $1,451(58.67 \%)$ \\
Electricity and Fuel Oil & $144(5.82 \%)$ \\
Electricity and LPG & $133(5.38 \%)$ \\
Electricity, Natural Gas and Fuel Oil & $14(0.57 \%)$ \\
Electricity, Natural Gas and LPG & $0(0.00 \%)$ \\
Electricity, Fuel Oil, and LPG & $22(0.89 \%)$ \\
Electricity, Natural Gas, Fuel Oil, and LPG & $0(0.00 \%)$ \\
Annual household expenditures (and consumptions) & \\
Electricity & $\$ 1,146(40.00 \mathrm{MBTU})$ \\
Natural gas & $\$ 856(78.16 \mathrm{MBTU})$ \\
Fuel oil & $\$ 1,736(118.61 \mathrm{MBTU})$ \\
LPG & $\$ 1,165(56.66 \mathrm{MBTU})$ \\
Outside good & $\$ 47,801$ \\
Unit prices (averaged over all Census regions) & \\
Electricity & $28.70 \$ / \mathrm{MBTU}$ \\
Natural gas & $10.94 \$ / \mathrm{MBTU}$ \\
Fuel oil & $14.74 \$ / \mathrm{MBTU}$ \\
LPG & $20.97 \$ / \mathrm{MBTU}$ \\
Household Attributes & \\
Annual household income & $\$ 49,654$ \\
Household with income below \$25k & $746(30.20 \%)$ \\
Household with income above $\$ 75 \mathrm{k}$ & $570(23.00 \%)$ \\
Household size (i.e., no. of people) & 2.68 \\
Age of householder & 50.01 years \\
Housing Unit Characteristics & \\
Age of housing unit & 38.59 years \\
Area of housing unit & 2,403 square feet \\
House in multifamily dwelling units & $407(16.46 \%)$ \\
Natural gas connection available to house & $1,779(71.94 \%)$ \\
Household Location Attributes & \\
House in rural locations & $491(19.85 \%)$ \\
House in Northeast Census region & $492(19.89 \%)$ \\
House in Midwest Census region & $593(23.98 \%)$ \\
House in South Census region & $744(30.08 \%)$ \\
House in West Census region & $644(26.04 \%)$ \\
Climate Variables & \\
Annual Heating Degree Days (HDD) & 4,399 \\
Annual Cooling Degree Days (CDD) & 1,407 \\
\hline & \\
\hline
\end{tabular}

\footnotetext{
a The reported annual household expenditures and consumptions were obtained by taking averages of expenditures and consumptions only for those households that consumed that energy type.
}

types) is indicative of the much higher percentage (100\%) of households spending a non-zero amount of their budget on electricity relative to other energy types. The effect of other variables are discussed by the variable group in the subsequent sections.

\subsubsection{Household characteristics}

Among the household characteristics, annual income is specified in a logarithmic form as well as using dummy variables representing low and high-income households (with medium income as the base for the dummy variable specification). The negative coefficient on the log (income) variable indicates that the proportion of household income (but not necessarily the absolute dollar amount) spent on energy consumption decreases with increasing income levels. This impact is further reinforced by the positive coefficient on the low-income dummy variable (though this dummy variable effect is only marginally significant). A policy implication of these results is that energy price increases can have a disproportionately higher impact on low-income households than other households (see Cashin and McGranahan, 2006). As low-income households might not be able to substantially reduce energy consumptions in response to price increases (since their energy consumption is likely for essential needs), increases in energy prices will compel them to spend more for their energy needs and leave them with less money to spend for other needs (Kilian, 2008). Moving on to the estimated coefficient on the high-income dummy variable, high-income households, in general, expend a higher share of their income (when compared to low-income households) on electricity. This is perhaps because high-income households tend to own and use a wider variety and a larger number of electric-operated appliances.

The household size (i.e., number of people in the household) related coefficients indicate that larger household sizes are associated with higher energy consumption in the context of electricity and natural gas. The log (householder age) variable refers to the natural logarithm of the age of the householder. The estimated effects of this variable imply that households at an older stage in their lifecycle are more pre-disposed to use non-electricity type of fuels (natural gas, fuel oil, and LPG) relative to electricity. 


\subsubsection{Housing unit characteristics}

The coefficients on the log (age of housing unit) variable indicate that households living in older houses are more likely to use fuel oil and natural gas than those living in newer houses. This result corresponding to fuel oil is consistent with the declining popularity of fuel oil as a heating source due to the extensive maintenance needs and environmental and health risks associated with fuel oil heaters and tanks. The result corresponding to natural gas is consistent with the trend that natural gas consumptions in the U.S. have declined over the years, perhaps due to two reasons (AGA, 2003): (1) the increased efficiency of natural gas appliances such as space heaters and water heaters, and better insulation features and energy efficiency of newer houses, and (2) the reduction in the number of gas appliances in homes served with gas.

As expected, households living in larger houses (in terms of house area) expend more on energy relative to households living in smaller houses. They are also more likely (than those in smaller houses) to use fuels other than electricity, such as natural gas, fuel oil, and LPG. It is likely that owners of smaller houses (with lower energy needs) are less likely to choose non-electricity types of fuels due to higher capital costs of installing equipment for such fuels as natural gas and fuel oil. On the other hand, as energy needs increase, it is likely that households choose fuels with lower prices and hence higher returns to offset the capital costs (see Mansur et al., 2008 for similar findings). Recall from Table 1 that the unit price of electricity is much higher than that of other fuels.

Households in multifamily units are associated with lower energy consumptions than those in single-family dwelling units and mobile homes. This may be attributable to the lower heating and other energy requirements due to such features as shared walls in multifamily units.

The final variable among housing unit characteristics corresponds to the availability of a natural gas pipe connection to the house. As indicated earlier, a household's choice between the natural gas and LPG alternatives depended on the availability of a natural gas pipe connection to its house. Further, no household chose both natural gas and LPG alternatives. Our model specification accommodates such perfect substitutability between the two alternatives by deterministically making the natural gas option available only to those houses with a natural gas pipe connection and the LPG option available only to those houses without a natural gas pipe connection. Besides such availability constraints in the context of natural gas and LPG fuels, the availability of a natural gas pipe connection also impacts the choice and consumption of other fuels. As expected, the presence of a natural gas pipeline decreases the energy expenditure on electricity and fuel oil.

\subsubsection{Household location characteristics}

Households in rural locations are less likely to choose natural gas but are more likely to choose LPG. This result may be due to the lower connectivity of natural gas utilities to rural locations. Also, households in southern regions appear to be less likely to opt for natural gas, while those in the north-eastern region are highly reliant upon fuel oil, a well-established trend (EIA, 2008).

\subsubsection{Climate variables}

The coefficients corresponding to the $\log$ (HDD) and log (CDD) variables indicate that households in colder climates expend more income share on energy than those in other climates, except for those households in very hot climates. These latter households expend more income share on electricity than households in other climates. These results are quite intuitive. Households in cold climates have increased space heating needs, leading to an increase in energy share expenditure in general and in non-electricity types of fuels in particular (space heaters generally use non-electricity sources of energy). On the other hand, households in very hot climates have space cooling needs, and air-conditioning units predominantly use electricity as the source of energy. In summary, the climate variable effects indicate that households in cold climates are more likely to use fuel oil, natural gas, and LPG, while those in hot climates tend to consume higher amounts of electricity. ${ }^{12}$

\subsubsection{Satiation and scale parameters}

The next set of parameters corresponds to the $\gamma_{k}$ terms. As discussed earlier, there is no such term corresponding to the electricity alternative because all households consume a non-zero amount of electricity (hence, no need for corner solutions). The $\gamma_{k}$ terms for other fuels are all significantly different from zero, indicating the possibility of corner solutions (i.e., zero consumption) for some households.

Finally, since there is variation in the unit prices $\left(p_{k}\right)$ across the energy/fuel choice alternatives, we could estimate the scale parameter associated with the Gumbel distribution of the baseline utility functions $\left(\psi_{k}\right)$ without assuming it to be unity (see Bhat, 2008). The scale parameter can be interpreted as a measure of the magnitude of unobserved factors influencing the baseline utility functions $\left(\psi_{k}\right)$. In a naïve MDCEV model with only constants in the baseline utility functions, and satiation parameters (i.e., a model with no other covariates), the scale parameter was estimated to be 0.608 (not shown in tables). As more and more explanatory

\footnotetext{
12 While the annual HDD and CDD variables are good measures of space heating and cooling needs, respectively, they do not unambiguously represent the seasonal temperature variations at a location. This is because the degree-days measure does not distinguish between a large number of moderately high/low temperatures and a small number of extremely high/low temperatures. Further, since the variables are constructed using $65^{\circ}$ Fahrenheit as the base temperature, there is an assumption that $65^{\circ}$ Fahrenheit is the optimum temperature at which least amount of energy is consumed. Using detailed information on temperature variations across different seasons in the model may overcome these limitations and provide better insights into climate effects on energy consumption. However, the RECS data does not contain such information. Further, at the least, countylevel geographic location information is required to be able to merge detailed temperature data from other sources into the RECS database. Mansur et al. (2008) include such detailed temperature information in their energy consumptions analysis.
} 
Table 2

MDCEV model estimation results of household energy consumption choices.

\begin{tabular}{|c|c|c|c|c|}
\hline Baseline utility function & Electricity & Natural Gas & Fuel Oil & LPG \\
\hline Baseline Preference Constants & $3.410(11.39)$ & $-0.398(-1.13)$ & $-10.214(-6.99)$ & $-2.392(-3.11)$ \\
\hline \multicolumn{5}{|l|}{ Household Characteristics } \\
\hline Log (Annual household Income) & $-0.954(-44.60)$ & $-0.954(-44.60)$ & $-0.954(-44.60)$ & $-0.954(-44.60)$ \\
\hline Dummy var. for low income $(<\$ 25 \mathrm{~K})$ & $0.068(1.58)$ & $0.068(1.58)$ & $0.068(1.58)$ & $0.068(1.58)$ \\
\hline Dummy var. for high income $(>\$ 75 \mathrm{~K})$ & $0.114(4.76)$ & - & - & - \\
\hline Household size & $0.111(15.29)$ & $0.040(4.07)$ & - & - \\
\hline Log (Age of householder) & - & $0.089(2.49)$ & $0.357(3.65)$ & $0.378(3.93)$ \\
\hline \multicolumn{5}{|l|}{ Housing unit Characteristics } \\
\hline Log (Age of housing unit) & - & $0.069(5.15)$ & $0.250(6.11)$ & - \\
\hline Log (Area of housing unit in sq. ft.) & $0.220(9.93)$ & $0.133(5.23)$ & $0.296(5.66)$ & $0.177(3.35)$ \\
\hline House is in a multifamily unit & $-0.194(-5.33)$ & $-0.194(-5.33)$ & $-0.194(-5.33)$ & $-0.194(-5.33)$ \\
\hline Natural gas connection available to house & $-0.448(-15.68)$ & - & $-0.714(-9.70)$ & - \\
\hline \multicolumn{5}{|l|}{ Household Location Characteristics } \\
\hline Rural location & - & $-0.250(-7.47)$ & - & $0.586(7.70)$ \\
\hline Southern census region & - & $-0.274(-9.65)$ & - & - \\
\hline North-Eastern census region & - & - & $0.899(10.63)$ & - \\
\hline \multicolumn{5}{|l|}{ Climate Variables } \\
\hline Log (Heating degree-days) & $0.046(3.28)$ & $0.152(7.15)$ & $0.813(5.51)$ & $0.169(2.81)$ \\
\hline Log (Cooling degree-days) & $0.133(14.20)$ & - & - & - \\
\hline Satiation (gamma) parameters & 0 (fixed) & $71.75(21.93)$ & $240.04(6.20)$ & $127.52(6.47)$ \\
\hline
\end{tabular}

Scale parameter $=0.331$, t-stat $=80$ against value of zero.

Log-likelihood at convergence $=-34,549.5$.

Log-likelihood at constants $=-36,823.5$.

variables were specified in the baseline utility functions, the magnitude of the scale parameter estimate decreased. In the final model specification (as shown in Table 2), the scale parameter estimate is 0.331 . The decrease in the magnitude of the scale parameter estimate from 0.608 for the naïve model to 0.331 for the final specification indicates that the specified explanatory variables capture about $46 \%$ of the variation in the logarithm of households' preferences toward different energy alternatives. This predictive value of the model is also corroborated by log-likelihood measures of fit. Specifically, the log-likelihood for the naïve MDCEV specification is $-36,823.5$, while that for the final MDCEV specification with 26 additional parameters is $-34,549.5$. The likelihood ratio statistic between the two specifications is 4,548 , which is substantially higher than the critical chi-square value for 26 degrees of freedom at any level of significance.

Overall, the MDCEV model estimates are consistent with expectations and shed light on the influence of different household, housing unit, location, and climate characteristics on residential energy consumption patterns. In the next section, we use these model estimates and the proposed forecasting procedure to examine various policy scenarios.

\section{Prediction experiments and scenario analysis}

In this section, we first present prediction experiments with the RECS data to assess the computational time performance of the proposed forecasting algorithms in the current empirical context (Section 6.1). For further assessment in choice situations with large choice sets, we present additional prediction experiments with simulated datasets in Section 6.2. Next, we conduct policy simulations to predict the impact of different climate change scenarios and other scenarios on residential energy consumption patterns in the U.S. (Section 6.3).

\subsection{Computational analysis of the proposed forecasting algorithms with small choice sets (with RECS data)}

To assess the computational performance of the proposed forecasting algorithms (presented in Sections 4.1 and 4.2 ), several prediction experiments were undertaken with different samples of the 4,382-household data, varying from 1,000 households to the full-RECS sample of 4,382 households. To recognize stochasticity, in each experiment, predictions were carried out repeatedly using several sets of draws of the $\varepsilon_{k}$ terms, varying from 100 sets to 500 sets for each household. Specifically, scrambled Halton sequences (Bhat 2003) of standard uniform distributions were generated and converted into Gumbel distributions with the scale parameter as estimated in the model and the location parameter as zero.

For all prediction exercises, both the proposed forecasting procedures from Sections 4.1 and 4.2 were coded and executed in the Gauss matrix programming language. In addition, for comparison purposes, the gradient-based constrained optimization routines of the Constrained Maximum Likelihood (CML) module of Gauss were used to undertake the same prediction exercises. ${ }^{13}$ Further, the numerical bisection algorithm of von Haefen et al. (2004) was also coded in Gauss and used to assess its performance vis-à-vis the

\footnotetext{
13 The CML module of Gauss uses a sequential programming method for constrained non-linear optimization, in which the optimal values are approximated iteratively using the gradients of the Lagrangian function.
} 
algorithms proposed in this paper and the gradient-based CML routines of Gauss. All predictions were performed with a desktop computer of $2.66 \mathrm{GHz}$ processing speed and 3.25 GB Random Access Memory (RAM).

Table 3 shows the computation times of the forecasting algorithms proposed in Sections 4.1 and 4.2 of this paper, as well as those of the von Haefen et al. algorithm and the gradient-based CML module of Gauss. In this table, the computation times reported in the first block of rows correspond to prediction runs of the incremental enumeration algorithm proposed in this paper for $\gamma$-profile utility functions (i.e., the algorithm proposed in Section 4.1) on datasets of different sample sizes and with different sets of error term draws. For example, to predict the energy expenditures/consumptions of 4,382 households, hundred different times for each household (each time with a different set of error term draws), the proposed incremental enumeration algorithm took only $10.62 \mathrm{~s}$ (see the last column). Another observation from the table is that the computation time increases almost linearly with the number of households (across the columns in each row) as well as with the number of sets of error term draws (across the rows in each column). Nevertheless, even for the entire dataset of 4,382 households, and with 500 sets of error term draws for each household, the total computation time is less than a minute.

The next set of rows in the table shows the computation times for the second forecasting algorithm proposed in this paper (in Section 4.2), which is designed for general utility functions that do not restrict the $\alpha_{k}$ parameters to be equal across alternatives. These computation times are at least 4 times longer than those of the algorithm designed specifically for $\gamma$-profile utility functions. The increase in computation time is because the general algorithm adopts an iterative approach to determine the value of the Lagrange multiplier $\lambda$, while the algorithm designed for $\gamma$-profile utility functions obviates the need for iterations by using an analytic expression for $\lambda$.

The third set of rows in the table shows the computation times for the von Haefen et al. (2004) algorithm. These computation times are about 14 times ( 3 times) longer than those of the algorithm proposed in this paper for $\gamma$-profile utility functions (more general utility functions). These run time differences highlight the efficiency of the algorithms proposed in this paper, especially the algorithm designed for $\gamma$-profile utility functions.

The final block of Table 3 shows the computational performance of the gradients-based, iterative constrained optimization routine (available in the CML module of Gauss). As can be observed, to compute the expenditure patterns of 4,382 households for just one set of error term draws for each household, it took $3 \mathrm{~h}$ and $36 \mathrm{~min}$. A linear extrapolation to 100 sets of error term draws implies a rather large computation time of about 15 days. These long run times highlight the inefficiency of the gradient-based, iterative algorithm. To be sure, we could reduce the computation time of the gradient-based algorithm by easing its convergence criteria. But this resulted in suboptimal solutions for a large number of households. In fact, even at the convergence criteria that resulted in the reported computation times of 15 days, the predicted expenditures for some households were suboptimal. Specifically, non-negligible differences were found between the predicted expenditures of the gradients-based procedure and that of the proposed procedure for a small number of households. In such cases, the utility values corresponding to the predictions from the proposed algorithms were higher compared to that of the iterative procedure, indicating that the gradient-based algorithm resulted in suboptimal solutions. As the convergence criteria were tightened to improve the accuracy of the results (i.e., to obtain more optimal solutions), the computation time increased considerably. In all fairness to the gradient-based iterative algorithm, perhaps such a level of accuracy is not necessary for practical purposes. However, as the convergence criteria were eased to reduce the computation time to a day (as opposed to 15 days), the quality of the results deteriorated too much to be of practical value. Thus, there seems to be a clear trade-off between accuracy and computation time that the analyst has to make when using a gradient-based algorithm. Even after making the trade-off, the analyst is left with an undesirable amount of computation time (in the order of days) for just one prediction exercise (with 4,382 households and 100 sets of error term draws). The incremental enumeration algorithm we propose for the $\gamma$-profile utility functions, on the other hand, is analytic in nature and does not leave room for any approximations or inaccuracy, and is free from any trade-off between accuracy and computational efficiency. ${ }^{14}$ Finally, in certain (although a small number of) instances with tight convergence criteria, the gradient-based procedure ran into convergence problems, requiring forced termination and resulting in suboptimal solutions. On the other hand, both the algorithms proposed in this paper as well as the von Haefen et al. algorithm did not run into convergence issues.

To further demonstrate the computational efficiency of the proposed algorithms, we undertook predictions with different sets of bootstrapped parameter estimates (obtained based on the covariance matrix of the model estimates). For example, as discussed earlier, for the entire dataset of 4,382 households, with 100 sets of error term draws, but only one set of parameter estimates, the computation time was $10.62 \mathrm{~s}$ for the incremental enumeration algorithm we designed (in Section 4.1) for $\gamma$-profile utility functions. When predictions were carried out with the same dataset and the same number of error term draws, but with 50 sets of bootstrapped parameter estimates, the computation time was about 8 and half minutes (510 s). With 100 sets of bootstrapped parameter estimates, the computation time was about $17 \mathrm{~min}(1,019 \mathrm{~s})$, which is not an unreasonable amount of time. To do the same, the other algorithm proposed in this paper (for more general utility functions; Section 4.2) took about 38 min for 50 bootstrapping repetitions and 76 min for 100 bootstrap repetitions. The von Haefen et al. algorithm took about $2 \mathrm{~h}$ for 50 bootstrapping repetitions and $4 \mathrm{~h}$ for $100 \mathrm{bootstrap}$ repetitions. On the other hand, the gradient-based, iterative algorithm would need an unreasonably large amount of time to do the same (it would need more than a year with 50 sets of bootstrapped estimates!).

\footnotetext{
14 The other algorithm we propose (for general utility functions) as well as the von Haefen et al. algorithm are also associated with similar tradeoffs between accuracy and computational efficiency, because of the iterative nature of these algorithms. For example, the von Haefen et al. algorithm accuracy could be increased in the decimal places of the predicted expenditures by tightening the convergence criteria that increased the run times to one-and-half times those reported in Table 3.
} 
Table 3

Run Times of the Proposed and Existing Forecasting Algorithms.

\begin{tabular}{|c|c|c|c|c|c|}
\hline \multicolumn{6}{|c|}{ Proposed algorithm for $\gamma$-profile utility functions (Section 4.1) } \\
\hline & 1,000 households & 2,000 households & 3,000 households & 4,000 households & 4,382 households \\
\hline \multicolumn{6}{|c|}{ \# Error draws } \\
\hline 100 sets & $2.37 \mathrm{~s}$ & $4.67 \mathrm{~s}$ & $7.05 \mathrm{~s}$ & $9.50 \mathrm{~s}$ & $10.62 \mathrm{~s}$ \\
\hline 500 sets & $11.49 \mathrm{~s}$ & $23.01 \mathrm{~s}$ & $34.71 \mathrm{~s}$ & $45.92 \mathrm{~s}$ & $50.77 \mathrm{~s}$ \\
\hline \multicolumn{6}{|c|}{ Proposed algorithm for general utility functions (Section 4.2) } \\
\hline & 1,000 households & 2,000 households & 3,000 households & 4,000 households & 4,382 households \\
\hline \multicolumn{6}{|c|}{ \# Error draws } \\
\hline 100 sets & $10.36 \mathrm{~s}$ & $20.86 \mathrm{~s}$ & $31.06 \mathrm{~s}$ & $41.63 \mathrm{~s}$ & $45.71 \mathrm{~s}$ \\
\hline 500 sets & $51.58 \mathrm{~s}$ & $104.17 \mathrm{~s}$ & $153.72 \mathrm{~s}$ & $204.39 \mathrm{~s}$ & $230.38 \mathrm{~s}$ \\
\hline \multicolumn{6}{|c|}{ von Haefen et al. (2004) algorithm } \\
\hline & 1,000 households & 2,000 households & 3,000 households & 4,000 households & 4,382 households \\
\hline \multicolumn{6}{|c|}{ \# Error draws } \\
\hline 100 sets & $32.80 \mathrm{~s}$ & $65.60 \mathrm{~s}$ & $98.51 \mathrm{~s}$ & $130.84 \mathrm{~s}$ & $143.45 \mathrm{~s}$ \\
\hline 500 sets & $163.56 \mathrm{~s}$ & $326.70 \mathrm{~s}$ & $491.42 \mathrm{~s}$ & $651.66 \mathrm{~s}$ & $717.69 \mathrm{~s}$ \\
\hline \multicolumn{6}{|c|}{ Gradient-based constrained optimization routines of Gauss } \\
\hline & 1,000 households & 2,000 households & 3,000 households & 4,000 households & 4,382 households \\
\hline \multicolumn{6}{|c|}{ \# Error draws } \\
\hline 1 set & $3,333 \mathrm{~s}$ & $6,237 \mathrm{~s}$ & $9,031 \mathrm{~s}$ & $11,876 \mathrm{~s}$ & $12,965 \mathrm{~s}$ \\
\hline
\end{tabular}

Note: Run times are on a desktop computer of $2.66 \mathrm{GHz}$ processing speed and 3.25 GB Random Access Memory (RAM).

It can be observed that the ratios of the above-reported run-times are similar to the ratios discussed earlier in this section. That is, the proposed incremental enumeration algorithm for $\gamma$-profile utility functions is about four times faster than the proposed algorithm in Section 4.2 (incremental enumeration combined with bisection over $\lambda$-space), and about 14 times faster than the von Haefen et al. algorithm. Similarly, the proposed algorithm in Section 4.2 is at least three times faster than the von Haefen et al. algorithm. Such computational efficiencies become crucial in application contexts with a large number of agents and when bootstrapping is needed to compute standard errors of the predictions.

\subsection{Analysis of the proposed forecasting algorithms in situations with large choice sets}

Admittedly, the above section demonstrates the computational efficiencies of the proposed forecasting algorithms in a choice situation with a small choice set. It is not clear if such computational efficiencies translate to situations with large choice sets. Thus, to evaluate the computational performance of the proposed algorithms in situations with large choice sets, we conducted simulation experiments for a 211-alternative choice set case with 1,000 observations. In these experiments, the utility function specification was a $\gamma$-profile similar to that in Equation (20), but with a single outside good. The deterministic component of the log-baseline utility for the outside good was set to 1 for identification, and that for the remaining 210 inside goods was specified as a linear function of a normally distributed variable. All prices were assumed to be 1 . The budget was kept equal for all choice observations in each experiment but varied from 1 to 100,000 across different experiments to generate situations with small sets of chosen alternatives as well as large sets of chosen alternatives. Table 4 reports the computation times based on prediction runs using 50 sets of error draws for each of the 1,000 observations in the simulated data. As can be observed from the first two rows of the table, as the budget increases from 1 to 100,000 (from left to right of the table), the average number of chosen alternatives increases from 1.5 to 115 . The next set of rows report the computations for each of the forecasting algorithms, including the von Haefen et al. algorithm. Several observations can be made from these computation times, as discussed below.

First, the two algorithms proposed in Sections 4.1 and 4.3.1 for $\gamma$-profile utility functions are much faster than the other three algorithms designed for general utility functions (including the von Haefen et al. algorithm). This is because these two algorithms exploit the properties of $\gamma$-profile utility functions to use closed-form expressions for optimal consumptions. Second, between the two algorithms for $\gamma$-profile utility functions, incremental enumeration performs faster for situations with a small to moderate number of chosen alternatives, and bisection-based enumeration performs faster for situations with a large number (50 or more) of chosen alternatives. We noticed during the simulations that the bisection-based enumeration arrives at the chosen alternatives in about $6-8$ steps, regardless of the number of chosen alternatives. Accordingly, the corresponding computation times for this algorithm are similar across all choice situations (regardless of the number of chosen alternatives). Third, among the three algorithms for general utility functions, the incremental enumeration combined with bisection over the $\lambda$-space (as in Section 4.2) performs the best in situations with a small to moderate number (i.e., up to 25) of chosen alternatives. In situations with a large number (beyond 50) of chosen alternatives, the method of bisection over the $\lambda$-space or the von Haefen et al. method of bisection over the consumption space are preferable. Further, as speculated earlier, the bisection over $\lambda$-space and the bisection over consumption space are computationally similar (see the similar computation times between the last two rows).

Based on these findings, the following general guidelines may be useful for an analyst using KT demand models, especially in largescale micro-simulation models that simulate choices of a large number of agents: 
- Use the incremental enumeration method (proposed in Section 4.1) in data environments with $\gamma$-profile utility functions and a small to moderate number (up to 50) of chosen alternatives.

- Use the bisection-based enumeration method (proposed in Section 4.3.1) in data environments with $\gamma$-profile utility functions and a large (more than 50) number of chosen alternatives.

- Use incremental enumeration combined with bisection over the $\lambda$-space (as in Section 4.2) in data environments with $\alpha$-profile utility functions and a small to moderate number (up to 50) of chosen alternatives.

- Use either the proposed bisection over $\lambda$-space (as in Section 4.3.2) or von Haefen et al.'s bisection over consumption-space in data environments with $\alpha$-profile utility functions and a large number (more than 50 ) of chosen alternatives.

\subsection{Scenario analysis}

This section presents the results of several policy simulations to assess the impact of four different scenarios on residential energy consumption/expenditure patterns in the U.S. All the simulations presented in this section were carried out using the incremental enumeration algorithm proposed in Section 4.1. Two of the four scenarios are related to climate change: (1) A $450^{\circ}$ Fahrenheit increase in annual cooling degree days (CDD), and (2) A $450^{\circ}$ Fahrenheit increase in annual heating degree days (HDD). These scenarios can be viewed as a result of a $5^{\circ}$ Fahrenheit rise (for the first scenario) or a $5^{\circ}$ Fahrenheit drop (for the second scenario) in average daily temperature from $65^{\circ}$ for three months ( 90 days) in a year. The other two scenarios are related to the size of housing units in the U.S.: (1) A $10 \%$ increase in the floor area of all houses, and (2) a 10\% decrease in the floor area of all houses. The predictions were carried out for 50 sets of bootstrapped parameter estimates. For each set of parameter estimates, 100 sets of error term draws were used for each household to simulate unobserved heterogeneity. The prediction results are presented in Tables 5-7, each of which is discussed next.

Table 5 presents the predicted residential energy expenditures as well as the observed expenditures for the base case scenario (the standard errors of the predictions are in parenthesis and are obtained from bootstrapping). The first two numeric columns (under the label of "sample predictions") of the table show the predicted energy expenditures and consumptions, averaged over all households, all sets of bootstrapped model parameters, and all sets of error term draws, for the sample data set. The third and fourth numeric columns (under the label of "sample observations") show the observed average energy expenditures and consumptions in the sample data. For example, the model predicts that, on average, a household spends \$1 100 per annum to consume 38 Million British Thermal Units (MBTU) of electricity, while the observed average household expenditure on electricity is \$1 116 per annum (for 39 MBTU). It can be observed that the aggregate predictions are reasonably close to the observed energy expenditures and consumptions, except a notable discrepancy in the context of natural gas consumption (the model seems to be over-predicting natural gas consumption). The weights available in the data were used to expand the predicted (and observed) expenditures and consumptions to total annual energy expenditures and consumptions for all the 111.1 million housing units in the U.S. Such population totals are shown in the second half of Table 5, under "population predictions" and "population observations". Similar predictions were obtained for all the above-mentioned four scenarios, as discussed next.

Table 6 shows the predictions for the two climate change scenarios. The first two numeric columns of the table contain the predicted changes in residential energy expenditure and consumption patterns due to a $450^{\circ}$ Fahrenheit increase in annual CDD. Both average predicted changes and standard errors (obtained from bootstrapping) are reported in the table, with the standard errors in parentheses. According to these predictions, the overall residential electricity energy expenditure in the U.S. would increase by $\$ 5.581$ Billion due to a $450^{\circ}$ Fahrenheit increase in annual CDD. This is due to an increase in electricity consumption by 189.78 Trillion BTU. It can be observed that most of the increase in expenditures due to additional electricity consumption is drawn from the outside good, which includes other household expenditures and savings. These results indicate severe welfare impacts of climate warming.

The third and fourth numeric columns in Table 6 present the predicted changes in residential energy expenditure and consumption patterns due to a $450^{\circ}$ Fahrenheit increase in annual HDD. The model predictions indicate an overall increase in residential energy expenditures by $\$ 5.104$ Billion, most of which is due to increased consumption of natural gas (by 191.48 Trillion BTU) and fuel oil (by 116.06 Trillion BTU). This is an expected result because space heating requirements increase at cooler temperatures, and natural gas

Table 4

Computational performance of the proposed algorithms in situations with large choice sets (computation time for 1,000 observations, with 50 sets of error term draws for each observation).

\begin{tabular}{|c|c|c|c|c|c|c|}
\hline Budget & 1 & 10 & 100 & 1,000 & 10,000 & 100,000 \\
\hline Avg. no. of chosen alternatives (alts) & 1.5 alts & 10 alts & 25 alts & 49 alts & 80 alts & 115 alts \\
\hline & \multicolumn{6}{|c|}{ Computation Time in Seconds (for 1,000 observations, with 50 different sets of error term draws) } \\
\hline \multicolumn{7}{|l|}{ Algorithms for $\gamma$-profile Utility Functions } \\
\hline Incremental enumeration (Section 4.1) & $9.30 \mathrm{~s}$ & $10.04 \mathrm{~s}$ & $11.41 \mathrm{~s}$ & $13.86 \mathrm{~s}$ & $15.72 \mathrm{~s}$ & $18.21 \mathrm{~s}$ \\
\hline Bisection-based enumeration (Section 4.3.1) & $12.10 \mathrm{~s}$ & $12.03 \mathrm{~s}$ & $12.12 \mathrm{~s}$ & $12.30 \mathrm{~s}$ & $12.56 \mathrm{~s}$ & $12.90 \mathrm{~s}$ \\
\hline \multicolumn{7}{|l|}{$\begin{array}{l}\text { Algorithms for General Utility Functions (including } \\
\alpha \text {-profiles) }\end{array}$} \\
\hline Incremental enumeration $\&$ bisection over $\lambda$-space (4.2) & $12.84 \mathrm{~s}$ & $16.50 \mathrm{~s}$ & $25.46 \mathrm{~s}$ & $44.50 \mathrm{~s}$ & $75.72 \mathrm{~s}$ & $111.70 \mathrm{~s}$ \\
\hline Bisection over $\lambda$-space (4.3.2) & $20.55 \mathrm{~s}$ & $28.40 \mathrm{~s}$ & $36.75 \mathrm{~s}$ & $44.75 \mathrm{~s}$ & $54.14 \mathrm{~s}$ & $60.61 \mathrm{~s}$ \\
\hline Bisection over consumption space (von Haefen et al., 2004) & $22.70 \mathrm{~s}$ & $31.66 \mathrm{~s}$ & $38.11 \mathrm{~s}$ & $45.22 \mathrm{~s}$ & $54.39 \mathrm{~s}$ & $60.86 \mathrm{~s}$ \\
\hline
\end{tabular}

Note: Run times are on a desktop computer of $2.66 \mathrm{GHz}$ processing speed and 3.25 GB Random Access Memory (RAM). 
Table 5

Predicted and observed energy consumption patterns in the base case.

\begin{tabular}{|c|c|c|c|c|c|c|c|c|}
\hline & \multicolumn{2}{|c|}{ Sample Predictions } & \multicolumn{2}{|c|}{ Sample Observations } & \multicolumn{2}{|c|}{ Population Predictions } & \multicolumn{2}{|c|}{ Population Observations } \\
\hline & $\begin{array}{l}\text { Average } \\
\text { annual }\end{array}$ & $\begin{array}{l}\text { Average } \\
\text { annual }\end{array}$ & $\begin{array}{l}\text { Average } \\
\text { annual }\end{array}$ & $\begin{array}{l}\text { Average } \\
\text { annual }\end{array}$ & Total annual & Total Annual & Total Annual & Total Annual \\
\hline & $\begin{array}{l}\text { household } \\
\text { expenditure }\end{array}$ & $\begin{array}{l}\text { Household } \\
\text { consumption }\end{array}$ & $\begin{array}{l}\text { household } \\
\text { expenditure }\end{array}$ & $\begin{array}{l}\text { household } \\
\text { consumption }\end{array}$ & $\begin{array}{l}\text { expenditure in all } \\
\text { houses in the U.S. } \\
\text { (Billion \$) }\end{array}$ & $\begin{array}{l}\text { consumption in all } \\
\text { houses in the U.S. } \\
\text { (Trillion BTU) }\end{array}$ & $\begin{array}{l}\text { Expenditure in all } \\
\text { houses in the U.S. } \\
\text { (Billion \$) }\end{array}$ & $\begin{array}{l}\text { consumption in all } \\
\text { houses in the U.S. } \\
\text { (Trillion BTU) }\end{array}$ \\
\hline & $(\$)$ & (Million BTU) & $(\$)$ & (Million BTU) & & & & \\
\hline Outside good & $45,766(37.2)$ & - & 45,805 & - & $5,235.24(4.0)$ & - & $5,239.99$ & - \\
\hline Electricity & $1,100(17.2)$ & $38(0.6)$ & 1,116 & 39 & $123.16(2.0)$ & $4,314(68.3)$ & 124.66 & 4349 \\
\hline Natural Gas & $548(21.4)$ & $50(2.0)$ & 487 & 44 & $59.66(2.4)$ & $5,515(217.8)$ & 52.38 & 4793 \\
\hline Fuel Oil & $157(21.2)$ & $11(1.4)$ & 149 & 10 & $13.62(1.9)$ & $925(127.3)$ & 12.75 & 865 \\
\hline LPG & $84(10.7)$ & $4(0.5)$ & 98 & 5 & $9.11(1.1)$ & $436(55.3)$ & 11.00 & 524 \\
\hline
\end{tabular}

and fuel oil are primarily used for space heating purposes.

Table 7 presents the predicted energy expenditure and consumption changes for scenarios related to the size of houses in the U.S. Specifically, it is predicted that a $10 \%$ increase in the floor area of all houses in the U.S. leads to an increase in residential energy expenditures by $\$ 4.741$ Billion. On the other hand, if the houses were $10 \%$ smaller in size, the residential energy expenditures would decrease by $\$ 5.117$ Billion.

\section{Summary and conclusions}

We propose simple and computationally efficient forecasting algorithms for random utility maximization-based multiple discretecontinuous choice models with additively separable utility functions, such as the MDCEV model. These algorithms build on simple yet insightful, analytic explorations of the KKT conditions of optimality that shed new light on the properties of such models. For specific forms of the consumption utility function (i.e., $\gamma$-profile utility function), the algorithm obviates the need for iterative constrained optimization procedures. The non-iterative nature of the algorithm contributes significantly to its efficiency and accuracy. Even with more general utility functions that fall within the class of additively separable utility functions, we use the properties of the MDCEV model to design efficient (albeit iterative) forecasting algorithms. We also propose variants of the proposed algorithms that perform faster in situations with large choice sets. Further, although developed in the context of the MDCEV model, the proposed algorithms can easily be modified for use with other utility maximization based KT consumer demand model systems with additively separable

Table 6

Predicted energy consumption/expenditure changes in response to climate change scenarios.

\begin{tabular}{|c|c|c|c|c|}
\hline & \multicolumn{2}{|c|}{$450^{\circ}$ Fahrenheit Increase in Annual Cooling Degree Days } & \multicolumn{2}{|c|}{$450^{\circ}$ Fahrenheit Increase in Annual Heating Degree Days } \\
\hline & $\begin{array}{l}\text { Predicted changes in energy } \\
\text { expenditures (Billion \$) }\end{array}$ & $\begin{array}{l}\text { Predicted changes in energy } \\
\text { consumption (Trillion BTU) }\end{array}$ & $\begin{array}{l}\text { Predicted changes in energy } \\
\text { expenditures (Billion \$) }\end{array}$ & $\begin{array}{l}\text { Predicted changes in energy } \\
\text { consumption (Trillion BTU) }\end{array}$ \\
\hline & Average (Standard error) & Average (Standard error) & Average (Standard error) & Average (Standard error) \\
\hline $\begin{array}{r}\text { Outside } \\
\text { good }\end{array}$ & $-5.281(0.454)$ & - & $-5.104(0.591)$ & - \\
\hline Electricity & $5.581(0.478)$ & $189.78(16.31)$ & $0.900(0.267)$ & $32.23(9.42)$ \\
\hline Natural Gas & $-0.169(0.016)$ & $-15.72(1.44)$ & $2.079(0.213)$ & $191.48(19.63)$ \\
\hline Fuel Oil & $-0.074(0.010)$ & $-5.03(0.70)$ & $1.712(0.371)$ & $116.06(25.16)$ \\
\hline LPG & -0.057 (0.009) & $-2.70(0.41)$ & $0.413(0.134)$ & $18.92(6.25)$ \\
\hline
\end{tabular}

Table 7

Predicted energy consumption/expenditure changes in scenarios associated with house area sizes in the U.S.

\begin{tabular}{|c|c|c|c|c|}
\hline & \multicolumn{2}{|l|}{ House Area Increased by $10 \%$} & \multicolumn{2}{|l|}{ House Area Decreased by $10 \%$} \\
\hline & $\begin{array}{l}\text { Predicted changes in energy } \\
\text { expenditures (Billion \$) }\end{array}$ & $\begin{array}{l}\text { Predicted changes in energy } \\
\text { consumption (Trillion BTU) }\end{array}$ & $\begin{array}{l}\text { Predicted changes in energy } \\
\text { expenditures (Billion \$) }\end{array}$ & $\begin{array}{l}\text { Predicted changes in energy } \\
\text { consumption (Trillion BTU) }\end{array}$ \\
\hline & Average (Standard error) & Average (Standard error) & Average (Standard error) & Average (Standard error) \\
\hline $\begin{array}{l}\text { Outside } \\
\text { good }\end{array}$ & $-4.741(0.631)$ & - & $5.117(0.670)$ & - \\
\hline Electricity & $2.405(0.284)$ & 84.39 (9.97) & $-2.609(0.303)$ & $-91.55(10.63)$ \\
\hline Natural Gas & $1.204(0.271)$ & $110.93(24.97)$ & $-1.313(0.292)$ & $-120.97(26.84)$ \\
\hline Fuel Oil & $0.797(0.162)$ & $54.10(11.02)$ & $-0.836(0.165)$ & $-56.77(11.21)$ \\
\hline LPG & $0.335(0.117)$ & $15.89(5.55)$ & $-0.358(0.121)$ & $-17.00(5.77)$ \\
\hline
\end{tabular}


utility functions.

To demonstrate the effectiveness of the forecasting algorithms, we present an analysis of residential energy consumption patterns in the U.S. using the MDCEV model and its forecasting algorithm proposed in this paper. Household-level energy consumption data from the 2005 Residential Energy Consumption Survey (RECS) conducted by the Energy Information Administration (EIA) was utilized for this analysis. The MDCEV model estimates provide insights into the influence of household, house-related, residential location, and climatic factors on households' consumption patterns of different types of energy, including electricity, natural gas, fuel oil, and liquefied petroleum gas (LPG).

Prediction exercises with the proposed algorithms, a numerical bisection-based iterative algorithm proposed by von Haefen et al. (2004) and a general gradient-based iterative algorithm indicate the significant computational efficiency (as well as accuracy) of the proposed algorithms. Further prediction exercises on simulated data with large choice sets suggest the following general guidelines. (1) For $\gamma$-profile utility functions, incremental enumeration performs well in choice situations with a small to moderate number (up to 50 ) of chosen alternatives, while bisection-based enumeration performs well in choice situations with a large number (beyond 50) of chosen alternatives. (2) For $\alpha$-profile utility functions, incremental enumeration combined with bisection over the $\lambda$-space performs well in situations with a small to moderate number of chosen alternatives, while a simple bisection over the $\lambda$-space or over the consumption-space (as in von Haefen et al., 2004) performs well in situations with a large number of chosen alternatives.

The computational efficiencies of the proposed algorithms facilitated policy simulations to predict the impact of climate change and other scenarios on residential energy consumption patterns. The results indicate that a $450^{\circ}$ Fahrenheit increase in cooling degree days over a year can lead to a $\$ 5.581$ billion increase in residential expenditures on electricity. This is due to 189.78 Trillion BTU of additional electricity consumption. Similarly, a $450^{\circ}$ Fahrenheit increase in cooling degree days over a year can lead to a $\$ 5.104$ billion increase in residential energy expenditures, primarily due to increased consumption of natural gas and fuel oil. In the context of house sizes in the U.S., if the houses were $10 \%$ smaller (larger) in their floor area, the residential energy expenditures would decrease (increase) by $\$ 5.117$ Billion ( $\$ 4.741$ Billion).

The effectiveness of the proposed algorithms for forecasting and policy analysis with MDCEV and other KKT demand model systems should facilitate the increased use of these model systems in applied research and policy analysis, as evidenced by a large number of studies that cite earlier versions of this work. One of the proposed algorithms has been used in large-scale activity-based travel demand micro-simulation systems for predicting the vehicle ownership/usage and activity-participation and time-use patterns of individuals in areas such as Los Angeles (with a population of about 20 million individuals). Another useful application of this algorithm is that it can be used to quickly generate large sets of synthetic data for simulation experiments to understand the properties of MDCEV and other KT demand model systems. For example, since a fast and easy-to-use forecasting procedure is available, further research can be conducted to characterize the distributions of forecasts obtained from KT demand systems. Besides, one can conduct predictive validation assessments of the MDCEV approach for different empirical contexts (Sikder and Pinjari, 2013; Pinjari et al., 2016).

Future studies would benefit from explicitly accommodating the potential endogeneity of energy prices in energy consumption (due to block pricing). Another extension is to include gasoline consumption (for travel needs) in the analysis. Including temperature and other weather variables at a fine space and time resolution is another useful direction for extending the current empirical analysis. On the methodological front, there is an important need to develop similarly easy forecasting approaches for KT demand models that allow flexible, non-additive utility functions (see Palma and Hess, 2020 for a recent effort in this direction).

\section{Author Statement}

Abdul Rawoof Pinjari: Conceptualization, Methodology, Software, Formal analysis, Investigation, Validation, Resources, Data curation, Writing - original draft, Writing - review \& editing, Project administration. Chandra Bhat: Conceptualization, Writing review \& editing.

\section{Declaration of competing interest}

The authors do not have any conflicts of interest in the context of this paper.

\section{Acknowledgements}

Earlier versions of this paper were presented at the 2010 Transportation Research Board Annual Meeting (Washington, D.C.) and the 2010 Innovations in Travel Modeling (ITM) Conference (Tempe, Arizona). The authors appreciate the comments from the audience of both these conferences as well as many other researchers who utilized the proposed algorithms and the forecasting codes in the past decade. Anonymous reviewers provided helpful inputs on an earlier version of this paper.

\section{References}

Ahn, J., Jeong, G., Yeonbae, K., 2007. A forecast of household ownership and use of alternative fuel pevehicles: a multiple discrete-continuous choice approach. Energy Econ. 30 (5), 2091-2104.

AGA/American gas Association, 2003. Patterns in Residential Natural Gas Consumption, pp. 1997-2001.

Banks, J., Blundell, R.W., Lewbel, A., 1997. Quadratic Engel curves, indirect tax reform and welfare measurement. Rev. Econ. Stat. 79, 527-539.

Bhat, C.R., 2003. Simulation estimation of mixed discrete choice models using randomized and scrambled Halton sequences. Transport. Res. Part B 37 (9), $837-855$. 
Bhat, C.R., 2005. A multiple discrete-continuous extreme value model: formulation and application to discretionary time-use decisions. Transport. Res. Part B 39 (8), $679-707$.

Bhat, C.R., 2008. The multiple discrete-continuous extreme value (MDCEV) model: role of utility function parameters, identification considerations, and model extensions. Transport. Res. Part B 42 (3), 274-303.

Bhat, C.R., Castro, M., Khan, M., 2013. A new estimation approach for the multiple discrete-continuous probit (MDCP) choice model. Transport. Res. Part B 55, 1-22.

Bhat, C.R., Castro, M., Pinjari, A.R., 2015. Allowing for complementarity and rich substitution patterns in multiple discrete-continuous models. Transport. Res. Part B 81 (1), 59-77.

Bhat, C.R., Sen, S., Eluru, N., 2008. The impact of demographics, built environment attributes, vehicle characteristics, and gasoline prices on household vehicle holdings and use. Trans. Res. B 43 (1), 1-18.

Bhat, C.R., Mondal, A., Asmussen, K., Bhat, A.C., 2020. A multiple discrete extreme value choice model with grouped consumption data and unobserved budgets. Transport. Res. Part B 141, 122-196.

Bhattacharyya, S.C., Timilsina, G.R., 2009. Energy demand models for policy formulation: a comparative study of energy demand models. Dev. Res. Group World Bank. Working Paper No. 4866.

Calastri, C., Hess, S., Daly, A., Maness, M., Kowald, M., Axhausen, K., 2017. Modelling contact mode and frequency of interactions with social network members using the multiple discrete - continuous extreme value model. Transport. Res. Part C 76, 16-34.

Cashin, D.B., McGranahan, L., 2006. Household Energy Expenditures, 1982-2005. Chicago Fed Letter. Federal Reserve Bank of Chicago. June 2006.

Castro, M., Bhat, C.R., Pendyala, R.M., Jara-díaz, S.R., 2012. Accommodating multiple constraints in the multiple discrete-continuous extreme value (MDCEV) choice model. Transport. Res. Part B 46 (6), 729-743.

Christensen, L., Jorgenson, D., Lawrence, L., 1973. Transcendental logarithmic production frontiers. Rev. Econ. Stat. 55, 28-45.

Clements, M.P., Madlener, R., 1999. Seasonality, cointegration and forecasting UK residential energy demand. Scot. J. Polit. Econ. 46 (2), $185-206$.

Dale, L., Fujita, K.S., Lavín, F.V., Moezzi, M., Hanemann, M., Guerrero, S., Lutzenhiser, L., 2009. Price Impact on the Demand for Water and Energy in California Residences. California Climate Change Center. August 2009.

Deaton, A.S., Muellbauer, J.N.J., 1980. An almost ideal demand system. Am. Econ. Rev. 70, 312-326.

Dubin, J.A., McFadden, D.L., 1984. An econometric analysis of residential electric appliance holdings and consumption. Econometrica 52, $118-131$.

EIA, 2008. "Residential Heating Oil Prices: what Consumers Should Know" EIA Brochure. \#: DOE/EIA-X048, released December 2008, retrieved June 1, 2010 from the EIA Website.

EIA, 2009. Annual Energy Review 2008. Report Prepared by the Energy Information Administration, Office of Markets and End Use. U.S. Department of Energy, Washington DC. June 2009. www.eia.doe.gov/aer.

Enam, A., Konduri, K., Pinjari, A.R., Eluru, N., 2017. An integrated choice and latent variable model for multiple discrete continuous choice kernels: application exploring the association between day-level moods and discretionary activity engagement choices. Journal of Choice Modelling $26,80-100$.

Fang, H.A., 2008. A discrete-continuous model of households' vehicle choice and usage, with an application to the effects of residential density. Transport. Res. Part B $42(9), 736-758$.

Ferdous, N., Pinjari, A.R., Bhat, C.R., Pendyala, R.M., 2010. A comprehensive analysis of household transportation expenditures relative to other goods and services: an application to United States consumer expenditure data. Transportation 37 (3), 363-390.

Filippini, M., Pachauri, S., 2004. Elasticities of electricity demand in urban Indian households. Energy Pol. 32, $429-436$.

Habib, K.M.N., Miller, E.J., 2009. Modeling activity generation: a utility-based model for activity agenda formation. Transportmetrica 5 (1), 3-23.

Hanemann, W.M., 1978. A Methodological and Empirical Study of the Recreation Benefits from Water Quality Improvement. Ph.D. dissertation, Department of Economics, Harvard University.

Hanemann, W.M., 1984. Discrete/continuous models of consumer demand. Econometrica 52, 541-561.

Houthakker, H.S., 1951. Some calculations of electricity consumption in great britain. J. Roy. Stat. Soc. 114 (3), $351-371$.

Kim, J., Allenby, G.M., Rossi, P.E., 2002. Modeling consumer demand for variety. Market. Sci. 21, 229-250.

Madlener, R., 1996. Econometric analysis of residential energy demand: a survey. J. Energy Lit. 2 (2), 3-32.

Mansur, E.T., Mendelsohn, R., Morrison, W., 2008. Climate change adaptation: a study of fuel choice and consumption in the US energy sector. J. Environ. Econ. Manag. 55, 175-193.

Narayan, P.K., Smyth, R., 2005. The residential demand for electricity in Australia: an application of the bounds testing approach to cointegration. Energy Pol. 33, $467-474$.

Nesbakken, R., 2001. Energy consumption for space heating: a discrete-continuous approach. Scand. J. Econ. 103 (1), $165-184$.

Palma, D., Hess, S., 2020. Some Adaptations of Multiple Discrete-Continuous Extreme Value (MDCEV) Models for a Computationally Tractable Treatment of Complementarity and Substitution Effects, and Reduced Influence of Budget Effects. Choice Modelling Centre, University of Leeds, UK.

Pellegrini, A., Pinjari, A.R., Maggi, R., 2021. A multiple discrete continuous model of time use that accommodates non-additively separable utility functions along with time and monetary budget constraints. Transport. Res. Part A 144, 37-53.

Phaneuf, D.J., Kling, C.L., Herriges, J.A., 2000. Estimation and welfare calculations in a generalized corner solution model with an application to recreation demand. Rev. Econ. Stat. 82 (1), 83-92.

Pinjari, A.R., 2011. Generalized extreme value (GEV)-based error structures for multiple discrete-continuous choice models. Transport. Res. Part B 45 (3), 474-489.

Pinjari, A.R., Bhat, C.R., 2010. A multiple discrete-continuous nested extreme value model: formulation and application to non-worker activity time-use and timing behavior on weekdays. Transport. Res. Part B 44 (4), 562-583.

Pinjari, A.R., Augustin, B., Imani, V.S., Eluru, N., Pendyala, R.M., 2016. Stochastic frontier estimation of budgets for Kuhn-Tucker demand systems: application to activity time-use analysis. Transport. Res. Part A 88, 117-133.

Rajagopalan, B.S., Srinivasan, K.S., 2008. Integrating household-level mode choice and modal expenditure decisions in a developing country: multiple discretecontinuous extreme value model. Transport. Res. Rec. 41-51, 2076.

Rajagopalan, B.S., Pinjari, A.R., Bhat, C.R., 2009. A comprehensive model of worker's non-work activity time-use and timing behavior. Transport. Res. Rec. 2134, 51-62.

Satomura, T., Kim, J., Allenby, G.M., 2011. Multiple-constraint choice models with corner and interior solutions. Market. Sci. 30 (3), 481-490.

Saxena, S., Pinjari, A.R., Roy, A., Paleti, R., 2020. Multiple discrete-continuous choice models with bounds on consumptions. Trans. Res. Part A.

Sikder, S., Pinjari, A.R., 2013. The benefits of allowing heteroscedastic stochastic distributions in multiple discrete-continuous choice models. Journal of Choice Modelling 9 (1), 39-56.

Tapia, R.J., de Jong, G.C., Larranga, A.M., Cybis, H.B., 2020. Application of MDCEV to infrastructure planning in freight transport. Transport. Res. Part A 133, $255-271$.

Vaage, K., 2000. Heating technology and energy use: a discrete/continuous choice approach to Norwegian household energy demand. Energy Econ. 22, 649-666.

Van Nostrand, C., Sivaraman, V., Pinjari, A.R., 2013. Analysis of long-distance vacation travel demand in the United States: a multiple discrete-continuous choice framework. Transportation 40 (1), 151-171.

Vasquez-Lavin, F., Hanemann, M., 2009. Functional forms in discrete/continuous choice models with general corner solutions. In: Proceedings of the 1st International Choice Modeling Conference, Harrogate, March 2009.

von Haefen, R.H., 2003. Incorporating observed choice into the construction of welfare measures from random utility models. J. Environ. Econ. Manag. 45 (2), $145-165$.

von Haefen, R.H., Phaneuf, D.J., Parsons, G.R., 2004. Estimation and welfare analysis with large demand systems. J. Bus. Econ. Stat. 22 (2), $194-205$.

von Haefen, R.H., Phaneuf, D.J., 2005. Kuhn-tucker demand system Approaches to nonmarket valuation. In: Scarpa, R., Alberini, A.A. (Eds.), Applications of Simulation Methods in Environmental and Resource Economics. Springer.

Wales, T.J., Woodland, A.D., 1983. Estimation of consumer demand systems with binding non-negativity constraints. J. Econom. 21 (3), $263-285$. 